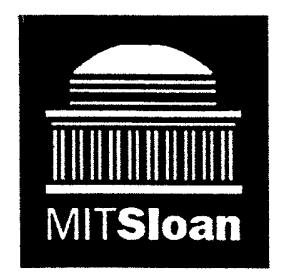

The International Center for Research on the Management of Technology

\title{
Internal Customers and Internal Suppliers
}

\author{
John R. Hauser \\ Duncan I. Simester* \\ Birger Wernerfelt
}

January 1995

WP \# 118-95

Revised June 1995

Sloan WP \# 3759

* University of Chicago, Graduate School of Business

(C) 1995 Massachusetts Institute of Technology

Sloan School of Management Massachusetts Institute of Technology

38 Memorial Drive, E56-390

Cambridge, MA 02139 


\section{ACKNOWLEDGMENT}

John R. Hauser is the Kirin Professor of Marketing, Massachusetts Institute of Technology, Sloan School of Management, 38 Memorial Drive, E56-314, Cambridge, MA 02139, (617) 253-2929, (617) 258-7597 fax. Duncan I. Simester is an Assistant Professor of Marketing, University of Chicago, Graduate School of Business, 1101 East 58th Street, Chicago, IL 60637. Birger Wernerfelt is a Professor of Marketing, Massachusetts Institute of Technology, Sloan School of Management, 38 Memorial Drive, E56-326, Cambridge, MA 02139.

This research was funded by the International Center for Research on the Management of Technology (ICRMOT). It has benefited from presentations before the member companies and, in particular, from a two-day ICRMOT workshop on the "Marketing/R\&D Interface" that was held at 3M. This paper has benefited from seminars at the Marketing Science Conference at the University of Arizona, M.I.T.,the University of Florida, the University of Minnesota, and the University of Pennsylvania. 


\begin{abstract}
In order to push a customer and market orientation deep into the organization many firms have adopted systems by which internal customers evaluate internal suppliers. The internal supplier receives a larger bonus for a higher evaluation. We examine such incentive systems and show that, under fairly general conditions, the internal supplier will normally share any gains with the internal customer in return for a higher evaluation. However, the firm, without loss of profit, can design incentive systems that anticipate this "gainsharing." We then examine two simple incentive systems. In one system the internal customer provides the evaluation implicitly by selecting the percentage of its bonus that is based on market outcomes (say a combination of net sales and customer satisfaction if these measures can be tied to incremental profits). The internal supplier's reward is based on the percentage that the internal customer chooses. In the second (different) system, the internal customer selects target market outcomes and the internal supplier is rewarded based on the target. For either system the firm can select parameters for the reward functions such that both the internal customer and the internal supplier choose the same profit-maximizing actions that the firm would choose if it had the power and knowledge to dictate actions, observe actions, and reimburse employees only for their costly actions. In each incentive system some risk is transferred from the firm to the employees and the firm must pay for this, but in return the firm need not observe either the internal supplier's actions or the internal customer's actions. The incentive systems are robust even if the firm guesses wrongly about what employees perceive as costly and about how employee actions affect profit. We discuss how these systems relate to internal customer satisfaction systems and to profit centers.
\end{abstract}


In order to drive customer satisfaction with our customers, IBM employees need to be satisfied with the organization and strive to exceed their own internal customer expectations.

-- Carder and Clark (1992, p. 29)

(Metropolitan Life Insurance Company of New York) developed a comprehensive program of measuring the expectation of all its customers, including both external and internal (employee) customers. ...only $25 \%$ (of the employees) are servicing the outside customer.

-- Zeithaml, Parasuraman, and Berry $(1990$, p. 58)

(At Weyerhaeuser) staff support departments such as human resources, accounting, and quality control have used "customer requirements analysis deployment" with line departments, such as sales, marketing, and branch production. ... (internal) customers are then asked to rate the suppliers ... in meeting each of their requirements.

-- McLaurin and Bell $(1993$, p. 38)

\section{Developing a Customer Orientation Throughout the Firm}

In the 1990s many firms believe that they will be more profitable if they can push a marketing orientation deep into the organization, particularly in new product development and in Research and Development (R\&D). In fact, these goals are the top listed and the top ranked research priorities of the Marketing Science Institute (1992-1994). Implementing a marketing orientation (including with employees and suppliers) remains one of MSI's three "capital" topics for 1994-1996. One aspect of this market orientation is to focus internal suppliers on serving their internal customer who, in turn, serves the (external) customers. To many firms, such internal suppliers are the next challenge in implementing a marketing orientation. The citations at the top of this page refer to IBM, Met Life, and Weyerhaeuser; other examples include 3M, ABB, Battelle, Berlex, Cable \& Wireless, Chevron, Corning, Hoechst Celanese, Kodak, Honda, and Xerox.' Marketing departments are often the internal customers of product development or R\&D, although in some cases Marketing is the internal supplier providing information on customer needs and requirements (Kern 1993). In most cases marketing professionals are called upon to help the firm develop a customer orientation for its internal suppliers. 
In many of these firms the internal customers evaluate the internal suppliers. For example, at an imaging firm the internal customers evaluate their internal suppliers on both short-term and long-term profit indicators. At an automobile parts firm the evaluations include measures that can be linked to the internal customer's ability to maximize the firm's profits. In some cases the internal supplier's compensation is tied directly to the evaluations; in other cases the tie is indirect with the evaluations used more qualitatively in job performance evaluation. Whether the compensation is explicit or implicit most internal suppliers recognize that, all else equal, they are more likely to be rewarded if they are evaluated well by the internal customers.

There are at least two reasons that motivate the internal customer-internal supplier evaluation systems. First, the goals of the internal suppliers may conflict with those of the firm. In addition to the usual problem that effort is costly to employees, employees may have different objectives than the firm. For example, one extensive study suggests that many R\&D scientists and engineers focus on publication and discovery of knowledge rather than on facilitating the ability of the firm (through the internal customers) to maximize profit (Allen and Katz 1992). In another example, Richardson (1985) suggests that $R \& D$ works on the technologies that they prefer rather than the technologies needed by the business areas. Furthermore, these conflicting objectives are not limited to R\&D groups (Finkelman and Goland 1990). For these situations, without incentives to the contrary, these internal suppliers would under-prioritize their customer's (and the firm's) concerns.

Secondly, the internal customer can often evaluate the impacts of the internal supplier's decisions while management may not have the skill, information, or time to do so as effectively. For example, Henke, Krachenberg and Lyons (1993) give an example of how an internal customer, the "interior trim" team, had better knowledge of how to solve a problem than did the overseeing product management team. This is especially true in $R \& D$ where the decisions often require specialized scientific or engineering knowledge not possessed by top managers. (In some cases top managers come from R\&D, but this is the exception rather than the rule.) Thus, top management direction or involvement is difficult at best. On the other hand, internal customers, such as marketing groups, who are affected by R\&D's decisions about where to direct its actions and efforts, can often evaluate R\&D better than top management.

A third factor, true in many but not all cases, is the significant time lags between the decisions by the internal supplier and market outcomes. For example, McDonough and Leifer (1986) suggest that planning and monitoring techniques rarely work for R\&D teams because 
commercial success is often not known for 5-10 years. In these cases it may be better to reward the internal supplier based on an internal customer's evaluation than it is to reward the internal supplier on market outcomes. (Although the time lag for the internal customer may be less than that for the internal supplier, it could still be significant. In this case the internal customer might, in turn, be rewarded based on an evaluation by its downstream customer. Alternatively the firm might choose to use other indicators that measure whether the internal customer is making the decisions that are best for the firm. See Hauser, Simester, and Wernerfelt 1994 for one example.)

In this paper we explore incentive systems in which the internal customer evaluates the internal supplier. We formulate the problem in terms of a marketing group as the internal customer and an R\&D group as the internal supplier. For example, the R\&D group might supply the technology that the marketing (or product development) group uses to develop a new product, or the R\&D group might supply a more-developed product which the marketing group must then sell to the external customer. Presumably the marketing group has more customer expertise than top management and, with the right incentives, it can use this expertise to provide an assessment of the ultimate customer valuation of the R\&D input. This example helps us illustrate the intuition but, hopefully, our analysis is not limited to these internal customer-internal supplier dyads and can be extended to situations where there are multiple internal suppliers and/or multiple internal customers. For example, one might also consider the marketing group (say a brand management group) as the internal supplier to the salesforce.

While such evaluation systems are popular, they are not always easy to implement. One issue is that internal customers may have a tendency to report favorably on their colleagues. In fact, internal suppliers might reward such behavior with various perks to the internal customers. Starcher (1992) gives an example where the internal supplier faced an aggressive goal to reduce the number of defects found by the internal customer. The internal customer found fewer faults, but only because it allowed more defects to be passed on to the final assembly group. This was costly to the firm because it required more rework. For many other examples, see Zettelmeyer and Hauser (1995). We show that in a broad class of internal customer-internal supplier systems such temptations toward collusion are inherent in internal customer-internal supplier incentive systems. However, we demonstrate that the firm can design incentive systems that take this tendency into account and perform well in spite of it. Specifically, we show that by allowing such cooperation (collusion) between the supplier and customer on the evaluation and by taking it into account, the 
firm can do at least as well as it could if it could costlessly prevent such cooperation.

The temptation for collusion on an evaluation will be greater if there is no cost to the internal customer for providing a higher evaluation. For example, if the internal customer just provides an evaluation on a 1-to-5 scale, if the internal supplier is told the evaluation it receives (by whom), and if management never questions any of the internal customer's evaluations, then Zettelmeyer and Hauser (1995) report many examples where internal customers give uniformly high evaluations. This temptation to provide high evaluations might be counteracted if there is some cost to the internal customer for providing a higher evaluation. This might be as simple as management questioning a history of "all 5's," it might take the form of management holding the internal customer to higher standards if the internal customer reports that it gets uniformly good input from its suppliers (i.e., gives all 5's), or it might be formalized. In this paper we consider reward systems in which it can be costly for the internal customer to give a higher evaluation.

We begin with fairly general reward functions to demonstrate the temptation for cooperation and to show how this temptation need not hurt the firm's profits. We then examine two specific reward systems. These systems share the properties that they use simple-to-specify reward functions and that they are relatively robust to errors that the firm might make in selecting the parameters of the reward functions. We are interested in such systems because they are more likely to be implemented than more complicated systems and are more likely to be implemented than systems in which the actions chosen by R\&D and Marketing are very sensitive to the parameters of the reward functions. While the two systems share many properties, they have distinct interpretations and thus provide two alternatives for the firms to choose.

The essential idea underlying both of the incentive schemes is that the internal customer need not evaluate the internal supplier by providing a written evaluation. It can reveal its evaluation of the internal customer(s) by selecting the parameters of its own reward function. ${ }^{2}$ For example, in one system the internal customer selects the percent of its compensation that depends on market outcomes (with the remaining percent based on a fixed bonus). In that system we interpret a larger percent as a higher evaluation of the internal supplier. That is, the internal supplier is doing its job better if the internal customer is more willing to risk market outcomes. In the other system the internal customer selects a target outcome (some combination of sales, satisfaction, incremental profits, etc.) In that system we interpret a higher target as a higher evaluation of the internal supplier. 
These systems are similar in many ways to systems in which the internal customer rates its satisfaction with the internal supplier, but they also differ in some ways. To make them equivalent the firm would have to make it costly, perhaps implicitly, for the internal customer to provide a higher rating. Specifically, in the first of the two systems, the firm would have to change the internal customer's rating to a percent and use that percent in the internal customer's incentive plan. Both the internal customer and the internal supplier would need to know this. In the second system, the firm would have to change a rating to a target and make the internal customer's bonus depend upon whether it achieves that target. Zettelmeyer and Hauser (1995) report that at least some $\mathrm{R} \& \mathrm{D} /$ business unit evaluation systems are beginning to look like one or the other of these two systems.

Our paper is structured as follows. We begin with a formalization of the problem. We quantify the tendency for the evaluatee (R\&D) to share its gains from the reward system with the evaluator (Marketing) and show when such sharing will normally occur. We then analyze the two simple schemes. We show that these schemes provide incentives to both Marketing and R\&D such that, acting in their own best interests, each chooses the actions that the firm would choose to maximize firm profits if it had the information and ability to do so directly and had to reimburse employees only for their costly actions. Because the ultimate market outcomes are uncertain and because these outcomes are only an indicator of employee actions, the firm must pay a risk-penalty to the employee groups. This risk penalty is a result of the uncertainty that the reward schemes impose on these groups. We quantify this penalty. Because we seek schemes that are implementable, we analyze their robustness in light of mistakes the firm might make. Throughout the exposition we interpret the formal model in light of our experience with internal customer-internal supplier systems. We close the paper by discussing how these incentive schemes relate to profit centers, management by objectives, and "quality tools."

\section{A Formal Model}

We consider two employee groups and one group of external customers. This will suffice to illustrate the basic points. For simplicity we call the internal supplier "Research and Development," label it with $R$, and refer to it as the upstream employee group. We call the internal customer "Marketing," label it with $M$, and refer to it as the downstream group. See Figure 1. 
$\underline{\text { Notation }}$

Research and Development $(R)$ expends "effort," $r$. This "effort" refers to the time and energy the R\&D group expends to identify, discover, or improve technology that Marketing, in turn, uses to develop products for customers. "Effort" also refers to decisions that $R$ might make that it views as costly because the decisions conflict with $R$ 's personal objectives. This effort is incremental above and beyond the effort $R$ must allocate in the absence of an internal customer-internal supplier incentive system. It is important to think of $r$ as costly effort. $R$ may work long hours, but if part of the time is on-the-job consumption that conflicts with the needs of the firm, then $r$ may be less than the long hours would suggest. For example, the Allen and Katz (1992) and the Richardson (1985) citations (and our own experience) suggest that $R$ prefers those technologies that are new, interesting, and lead to peer recognition and patents. These technologies may conflict with the needs of the internal customer. $R$ 's efforts, $r$, might include the time and energy necessary to understand the needs of Marketing beyond that which $R$ would otherwise allocate. We represent the perceived costs to $R$ as $c_{R}(r)$, where $c_{R}$ is thrice differentiable, increasing, and convex. Because the costs are incremental we normalize $c_{R}(0)=0$. Formally we assume that after $R$ chooses and expends $r, M$ can observe $r$ but top management (the firm) cannot. For example, consider a situation drawn from our experience with the research and marketing divisions at a major oil company. $R$ was working on the problem of getting more information to $M$ from remote oil fields. In this situation $M$ (but not top management) might be able to evaluate whether $R$ 's new data compression algorithm allows enough information to be transferred so that $M$ can meet its customer's needs.

Marketing $(M)$ uses the technology that $R$ develops and expends its own incremental effort, $m$, to serve the customers. We define $m$ to represent incremental, costly efforts, actions, and decisions. (Henceforth, we simply call $m$, "efforts.") We represent the perceived costs to $M$ as $c_{M}(m)$, where $c_{M}$ is thrice differentiable, increasing, and convex. We normalize $c_{M}(0)=0$. If $R$ expends more effort, $r$, then $M$ finds that its own efforts are more effective as defined next. For example, a better data compression algorithm might enable $M$ to provide better service to its customers. However, $M$ must also expend costly effort to provide that better service. The firm does not observe $m$ directly.

We assume that the firm observes an indicator of the profits that it obtains from the actions 
of $R$ and $M$. It uses this profit measure as a (noisy) indicator of $r$ and $m$. In our example, the firm might observe the increase in profits (more oil recovered, reduced costs) due to the new data transfer system. That is, the firm might compare the profits it now obtains versus those it would have obtained with the old data transfer system. (Here we assume the firm can account for other impacts on the profitability of the remote oil field.)

In practice, this profit indicator can take many forms. Zettelmeyer and Hauser (1995) report that one firm uses measures of quality, cost-effectiveness, timeliness, communications, and satisfaction from the (external) customer as an indicator of "profits" from $r$ and $m$. They also report that another firm uses downstream production cost, labor cost, quality cost, and production investments as indicators of the effect or $r$ and $m$ on short- and long-term profit. If we are to use these measures as proxies for incremental profit, we must assume that the $r$ and $m$ that maximize these indicators (net of cost) are the same values of $r$ and $m$ that maximize incremental profit. Hauser, Simester and Wernerfelt (1994) provide another example. They demonstrate that if the internal customer maximizes a weighted sum of (external) customer satisfaction and sales (net of costs) then the internal customer chooses the "efforts" that maximize the firm's long-term profits. In their case we would use a weighted sum of satisfaction and sales (net of costs) as a proxy for the incremental profits due to $r$ and $m$. See also Anderson, Fornell and Lehmann (1994). For the purposes of this paper we only need that the firm can observe some measure that indicates the incremental impact of $r$ and $m$ on the firm's profits. For simplicity we call this outcome measure profits, $\check{\pi}(r, m)$. We assume that the firm can scale the measure (or combination of measures) in the units of currency so that it represents the incremental contribution to profits from $R$ and $M$.

Because this measure (or combination of measures) may not always equal true accounting profits, we must model the error introduced by the use of this measure. We write this measure as equal to its mean, $\pi(r, m)$, plus zero-mean, normally distributed noise, ${ }^{3} e$. That is,

$$
\check{\pi}(r, m)=\pi(r, m)+e \quad \text { where } \quad e \sim N\left(0, \sigma^{2}\right)
$$

$\pi$ is thrice differentiable, increasing, and concave in both arguments; $\sigma^{2}>0$. We model the riskneutral firm as using the expected value of $\check{\pi}$ in the profit-maximization equation that relates to $R$ and M. (The expected value is $\pi$.)

After observing $r, M$ chooses an evaluation, $s$, that indicates to the firm how it values $r$. (We 
will later use $s_{1}$ and $s_{2}$ to distinguish between the two reward systems we analyze.) We use the mnemonic, $s$, because we think of this evaluation as an indicator of how well the internal supplier $\underline{\underline{s}}$ atisfies the internal customer. However, $s$ may not be measured on a typical satisfaction scale. In one of our reward systems it is a percent; in the other a target outcome for $\check{\pi}$. In both of these reward systems $M$ simply chooses the parameters of its own reward function (bonus plan) and the firm infers $s$ from $M$ 's choice of reward functions.

$M$ chooses $s$ before selecting $m$, but $M$ will anticipate how it will set $m$. That is, Marketing evaluates R\&D and will do so anticipating how it will use R\&D's output to serve Marketing's customers. For example, $M$ might choose its bonus plan, and hence evaluate $R$, after observing a demonstration of the data compression algorithm. $M$ would do so anticipating how it would use that algorithm to serve its customers and it would do so knowing that $s$ affects its own rewards. (Technically, we could also have stated the sequence as $M$ choosing $s$ simultaneously with $m$ because no one except $M$ observes $m$ directly.)

After observing $s$ the firm gives a bonus, $v$, to $R$ that depends on $s$. We write this function as $v(s)$. At a later time the firm observes the profit measure, $\check{\pi}$, and provides a bonus, $w$, to $M$ that depends upon this measure and on $M$ 's choice of $s$. We write this function as $w(s, \check{\pi})$. We restrict our attention to incentive systems with integrable and thrice differentiable ${ }^{4} v$ and $w$ which are concave in $s$. In keeping with the managerial statement of the problem we consider rewards to $R$ that are larger for higher implicit evaluations (increasing in $s$ ). We also want $s$ to be an indicator of $r$ 's effect on $\pi$, thus we restrict our attention to $w$ such that $\partial w^{2} / \partial r \partial s>0$.

It is convenient to think of $v$ and $w$ as monetary rewards; however, they need not be. Any set of rewards that $R$ and $M$ value and for which the firm must pay would be appropriate including new equipment, training, recognition, and awards (Feldman 1992, Mitsch 1990). For simplicity we assume that the amount that the firm pays is equal to the value that the employee group receives.

We assume that the firm is risk neutral and profit maximizing and that both $R$ and $M$ will act in their own best interests to maximize their expected utilities. We assume that both $R$ and $M$ are risk averse and that perceived costs to $R$ and $M$ are measured on the same scale as rewards. ${ }^{5}$ The utilities, $U_{R}$ and $U_{M}$, are:

$$
\begin{aligned}
U_{R}(s, r) & =U_{R}\left[v(s)-c_{R}(r)\right] \\
U_{M}(s, \check{\pi}, m) & =U_{M}\left[w(s, \check{\pi})-c_{M}(m)\right]
\end{aligned}
$$


where $U_{R}$ and $U_{M}$ are integrable, thrice differentiable, increasing, and concave.

We assume that the net utilities, $\bar{U}_{R}$ and $\bar{U}_{M}$, required by $R$ and $M$ in order to participate are set by the market -- that is, by the other options that $R$ and $M$ have available. (If there are any switching costs favoring the firm, then these are included in the definition of $\bar{U}_{R}$ and $\bar{U}_{M}$.) Thus, the total expected utility of $R$ 's and $M$ 's rewards minus their costs for allocating $r$ and $m$ and reporting $s$ must exceed $\bar{U}_{R}$ and $\bar{U}_{M}$. We normalize the utility functions such that they imply that (riskless) market options for $R$ and $M$ are equal to zero. (If the market options have risk, then they must be such that $R$ and $M$ consider them equivalent to a riskless option that is scaled to zero.) In its maximization of expected profits, a risk neutral firm will attempt to set the expected utility to each employee group just above $\bar{U}_{R}$ or $\bar{U}_{M}$ if, by doing so, it can earn non-negative profits. In our example, this means that the oil firm must select $v$ and $w$ such that $R$ and $M$ are willing to develop and use a new data compression algorithm rather than continue to serve the customer with the old system. Recall that the practical problem requires that the oil firm do this without knowing the technical details of compression algorithms.

The following summarizes the sequence of moves described so far.

$\checkmark \quad$ The firm announces an internal customer-internal supplier incentive system, $v(s)$ and $w(s, \pi)$.

$\checkmark \quad R$ chooses its actions, $r$, or does not participate.

$\checkmark \quad M$ observes $R$ 's actions, but the firm does not.

$\checkmark \quad M$ chooses $s$ or does not participate. ( $M$ might choose $s$ implicitly by selecting the parameters of its bonus system.)

$\checkmark \quad R$ is rewarded based on $v(s)$.

$\checkmark \quad M$ chooses its actions, $m$, but the firm can not observe these actions.

$\checkmark \quad$ The profit indicator, $\check{\pi}$, is observed and the firm pays $w(s, \check{\pi})$.

This sequence of moves is a well-defined contracting problem and we could, in principle, evaluate the performance of alternative $v$ 's and $w$ 's. In this contracting problem, with noise and risk aversion, very simple contracts will not do well. The firm can do better by tying pay to performance than by just paying a fixed salary. However, we will show that in some cases (Proposition 3), but not all cases (Proposition 4), the internal customer-internal supplier evaluation systems can be interpreted as a means to implement a system where $M$ 's pay depends upon performance and $R$ 
receives a bonus if and only if $M$ decides to participate optimally. In this case, we will interpret an internal customer-internal supplier evaluation system as a means to transfer responsibility for incremental profits from the firm to the internal customer, $M$. In the language of agency theory, this special case makes " $M$ a residual claimant on $\check{\pi}$."

In the formal contracting problem we focus on one set of actions, $r$ and $m$. In practice the firm would not reset $v$ and $w$ for every decision that $R$ and $M$ must make. The firm might set $v$ and $w$ such that they apply to repeated interactions between $R$ and $M$. We do not solve this problem formally. However, if we favor incentive systems in which the parameters of $v$ and $w$ are robust with respect to the specifics of $\pi, c_{M}$, and $c_{R}$, then it is more likely that the key parameters of $v$ and $w$ do not need to be set for every interaction. We formalize this robustness issue in a later section.

Before we go on, there is an additional complication that we must consider. If $R$ is rewarded based on $M$ 's choice of $s, R$ and $M$ might cooperate in the selection of $s$. (Internal customers might have more opportunities to interact with internal suppliers than do outside customers.)

\section{Will $M$ and $R$ Cooperate in the Choice of the Evaluation, $s$ ?}

It is reasonable to assume that marketing and $R \& D$ groups will find ways to cooperate, either explicitly or implicitly, on $s$. That is, $R$ could "pay" $M$ to provide an evaluation, $s$, that maximizes their combined utilities. $M$ would provide a higher $s$ if it were paid by $R$ at least as much as it loses because the new $s$ no longer maximizes $w(s, \pi) . R$ would pay $M$ if it received at least as much from the new $s$ [through $v(s)$ ] as it paid to $M$. Recent trends in total quality management and in R\&D management include many mechanisms to enhance communication and cooperation between Marketing and R\&D (Griffin and Hauser 1994). These mechanisms could enhance the ability to cooperate on $s$. We follow the Total Quality Management literature and call such cooperation "gainsharing." We prefer the more positive word, "gainsharing," to the pejorative words of "collusion" or "bribery," in part, because we analyze systems in which this cooperation on $s$ does not hurt either the customer or the firm. Our choice of words is consistent with the idea that the firm gets a higher $\pi$ and shares these gains with $R$ based on $s$. $R$ shares these gains with $M$.

Certainly the ability of $R$ and $M$ to cooperate on $s$ depends upon there being a small number of evaluators for any given evaluatee. (In figure 1 we abstract to one evaluator, but the qualitative 
ideas are easily extended to a few evaluators.) If the number of evaluators is very large, say hundreds, then it is likely to be too costly for the evaluatee to seek out every evaluator. In such cases it is also likely that the cost imposed on the evaluator for providing a higher evaluation would be small. Systems with large numbers of evaluators look more like a traditional (external) customer satisfaction systems than the internal customer-internal supplier systems that we analyze here.

We illustrate the concept of cooperation with an example between a sales person and the external customer. A colleague recently purchased an automobile. As part of the delivery transaction, our colleague was asked to complete a customer-satisfaction survey. He did so to the best of his ability. After looking at the customer's ratings, the salesperson said that the ratings were unacceptable and that he would be fired if the ratings were not increased. Our colleague agreed to increase the ratings, but in return for a year's free routine maintenance for which the salesperson paid. The salesperson agreed and the ratings were increased. (We presume that the cost of the routine maintenance was less than or equal to the bonus the salesperson received for the higher ratings. The dealership may or may not have known about this practice and may or may not have taken it into consideration in setting the salesperson's bonus system.) More recently, one of us purchased a new car. Not only did the sales manager instruct the customer on how to complete the customer-satisfaction questionnaire and imply that his access to supply depends upon the ratings, but he sent the customer an expensive gift the day prior to the completion of the satisfaction questionnaire. (We were told that the manufacturer allocated supply of this popular car to dealerships based on the ratings. Presumably the dealership found it more efficient to increase customer satisfaction with this gift than with other forms of service. Certainly, the customer, one of us, was satisfied.)

It might be difficult for an outside observer to document gainsharing between internal suppliers and internal customers. However, managers (and reward systems consultants) have indicated to us that they believe that modest gainsharing is common. This gainsharing usually takes the form of $R$ providing extra-normal service and perquisites to $M .^{6}$

A classic example of gainsharing is provided by Gouldner's (1965) account of a small gypsum mine. Gouldner describes a situation in which workers and managers lived as friends and neighbors in a small rural community. There arose an "indulgence pattern" whereby workers were allowed a great deal of discretion at work while management could maintain friendly relations with them outside the mine. The pattern collapsed when a new top manager, an outsider, put an end to 
the indulgence pattern. The miners struck for higher wages. Sidrys and Jakštaitè (1994) document an example of gainsharing in Lithuania where universities pay student stipends based on grades, local instructors are paid very low wages, and students pay instructors for higher grades. Perhaps the best known form of gainsharing is frequent flyer programs where airlines provide free leisure travel directly to customers who choose the airlines for business travel. This system may even be efficient. When IBM tried to negotiate with United Airlines to eliminate frequent flyer credits in return for fare discounts, United Airlines refused. A Boston Globe editorial (March 13, 1994) applauded frequent flyer programs as effective compensation for "the working stiff" and opined that the increase in the morale of IBM's employees from the programs is greater than the potential savings to IBM from a fare discount. The editorial ended with the opinion that "American productivity will be better for it."

We now analyze gainsharing with the formal model. To simplify the analysis of gainsharing we follow Tirole (1986) and assume that $R$ and $M$ will find a way to make a binding agreement exchanging a gainshare of goods or services valued at $g$ in return for a higher evaluation. The enforceability of the agreement could come from expected future interactions between $R$ and $M$ or from social norms. (For example, in the Sidrys and Jakštaitè [1994] data, gainsharing occurs more often with local instructors than with foreign instructors.) In the gainsharing agreement we note that the assumption is that $R$ and $M$ can cooperate on $s$, but not on $r$ and that the payment, $g$, cannot be contingent on $\check{\pi}$. In our oil company example, this assumption implies that it is easier for $R$ and $M$ to cooperate on $M$ 's evaluation of $R$ than it is for $R$ and $M$ to cooperate directly on the amount of "effort," $r$, that $R$ puts into developing the data compression algorithm. In situations were $R$ and $M$ can cooperate directly on $r$ as well as $s$, then this assumption restricts the domain of our analysis. However, we feel that this assumption is an important starting point and that it applies to most of the situations that we have observed. We have observed that it is much harder to monitor agreements about "average effort over a month (including detailed technology decisions)" than it is to monitor agreements about a single performance evaluation. We leave cooperation on $r$ to future research. Thus, formally, we augment the sequence of events such that $R$ and $M$ can make a binding agreement, $(g, s)$, after $M$ has observed $r$ but before $M$ has selected $s$. See Figure 2.

The gains (if any) from the agreement can be split in many ways between $R$ and $M$. To simplify the exposition we model the split as a take-in-or-leave it offer of $(g, s)$ from $R$ to $M$. This 
means that $M$ receives only as much as is necessary to induce $M$ to report the agreed-upon $s$. This assumption does not affect the qualitative interpretations. We could derive similar results for other sharing mechanisms.

We define $\hat{m}$ and $\hat{s}$ as the efforts and evaluation that $M$ will select to maximize $U_{M}$ for a given $r$ with no gainshare. For concave $U_{M}(\bullet)$, this maximization of expected utility by $M$ defines three continuously differentiable functions, $\hat{m}(r), \hat{s}(r)$, and $\hat{\pi}(r)$. That is, after $R$ selects $r$ these functions tell us the efforts and evaluation that $M$ would select if gainsharing were precluded. Suppose that for a given $r, R$ wants to influence $M$ to choose another $\tilde{s}$ (and the implied $\tilde{m}$ and $\tilde{\pi}$ ), more favorable to $R$. (That is, $\tilde{m}(r, \tilde{s})$ maximizes $M$ 's expected utility given $r$ and $\tilde{s}$. Note that $\tilde{m}$ may differ from $\hat{m}$ if $\tilde{s}$ differs from $\hat{s}$.) To do this, $R$ must give $M$ a gainshare, $g$, that at least compensates for $M$ 's loss. This means that $M$ 's expected utility with a gainshare-evaluation agreement, $(g, \tilde{s})$, must at least equal the expected utility that $M$ could obtain without accepting the gainshare. Thus, the minimum gainshare that $M$ will accept is defined by Equation 3 .

$$
E U_{M}\left[w(\tilde{s}, \tilde{\pi})-c_{M}(\tilde{m})+g\right]=E U_{M}\left[w(\hat{s}, \hat{\pi})-c_{M}(\hat{m})\right]
$$

$R$ has no incentive to give more than this $g$ in return for $\tilde{s}$, thus $R$ will attempt to get $g$ down to that defined in Equation 3 and $M$ will try to get $g$ up to that defined in Equation 3 . Thus, Equation 3 defines a critical value of $g$ for every $r$. We write this critical value as $g(r)$. $R$ wants to maximize its own well-being. That is, $R$ will select $\tilde{r}$ and $\tilde{s}$ to maximize its own expected utility:

$$
E U_{R}[\tilde{s}, \tilde{r}]=E U_{R}\left[v(\tilde{s})-c_{R}(\tilde{r})-g(\tilde{r})\right]
$$

where $g$ is implied by Equation 3 and $\tilde{m}, \hat{m}$, and $\hat{s}$ are implicit in $M$ 's maximization problems.

In summary, $R$ maximizes the expression in Equation 4 subject to the constraints imposed by the two maximization problems that define Equation 3. The right-hand side of Equation 3 describes what $M$ would do if there were no gainsharing and the left-hand side of Equation 3 describes what $M$ would do if gainsharing were allowed. Naturally, both sides of Equation 3 must be at least as large as that which $M$ could obtain by not participating. Equation 4 must be at least as large as that which $R$ could obtain by not participating. ( $R$ must consider $M$ 's participation because it can not get $\tilde{s}$ if $M$ does not participate.) The firm is interested in maximizing its profits so it will attempt to select $v$ and $w$ such that it gets the efforts it wants and it does not pay $R$ and $M$ 
more than is necessary. That is, Equations 3 and 4 will exceed the participation constraints by as little as possible. Once the firm specifies $v$ and $w$, these constraints and maximization problems are sufficient to solve for $\tilde{r}, \tilde{m}, \tilde{s}, \hat{m}, \hat{s}$. and the implied $\tilde{g}$ and $\tilde{\pi}$.

We now have the structure to demonstrate when there is temptation to cooperate on $g$ and $s$. We want the proposition to be as general as possible so we state the proposition both for the internal customer-internal supplier reward systems which constrain $s$ and for internal customerinternal supplier systems that do not constrain $s$. (For example, $s$ might be constrained if it is reported implicitly by $M$ as a percent or a rating. If it is reported by $M$ as a target outcome, then there may be no need to constrain $s$.)

PROPOSITION 1 (TEMPTATION TO GAINSHARE). For incentive systems in which the rewards to the internal supplier are increasing in the internal customer's evaluation, the internal supplier will be tempted to provide a positive gainshare to the internal customer (unless the firm sets a binding upper bound on the evaluation). Even with a binding upper bound, there may be temptation to gainshare.

The proof in the appendix handles the complexity of the optimization problems implied by Equations 3 and 4 . However, the idea of the proof is quite simple for interior solutions. Without a gainshare, $M$ will select an evaluation such that the marginal cost to $M$ of increasing the evaluation is zero at optimality $\left(\partial E U_{M} / \partial \hat{s}=0\right)$. However, the marginal gain to $R$ at this point is still positive because its rewards are increasing in $s$. Thus, there are gains to trade at $\hat{s}$.

The technical condition on a binding upper bound comes when the firm prevents $R$ and $M$ from selecting the $s$ that they would like to select (as implied by the unconstrained maximization problems). For example, suppose that $R$ and $M$ would like to select an $s$ equal to $100 \%$, but, by fiat, the firm announces that no $s$ can exceed $50 \%$. Under such conditions $R$ and $M$ may find that it is not worthwhile to agree on a gainshare. (Proposition 1 applies for all $v$ and $w$ that satisfy the stated conditions, not just for those $v$ and $w$ that are optimal for the firm.) Without specifying the reward functions, $v$ and $w$, further, we can not rule out the existence of a constrained reward scheme under which no gainsharing takes place. ${ }^{7}$ However, we provide an example in the next section where a binding upper bound is imposed and gainsharing does take place.

If the temptation to gainshare is inherent in the structure of internal customer-internal supplier 
incentive systems, then we would like to know how this affects the ability of the firm to maximize profits. In the following proposition we demonstrate that gainsharing does not impair the ability of the firm to select a $v$ and $w$ that maximize profits. The firm can do at least as well by allowing gainsharing as it can do by precluding gainsharing. (If it is costly to the firm to preclude gainsharing, then the firm can do better by allowing gainsharing.)

PROPOSITION 2 (GAINSHARING DOESN'T HURT PROFITS). If the firm can preclude gainsharing and design a reward system, $v$ and $w$, to implement $r$ and $m$, then, without any loss of profits, the firm can design a reward system that allows gainsharing and implements the same $r$ and $m$.

The proof in the appendix is by construction. We show that the firm can factor out gainsharing and implement the same actions with a new reward system which subtracts $\alpha s$ from $w$ and adds $\alpha s$ to $v$. Because the noise enters $\check{\pi}$ and the changes in the reward system do not depend upon $\check{\pi}$, the risk costs that $R$ and $M$ bear are unaffected. Thus, the same actions imply the same profits. (In the proof we show that an $\alpha$ exists that implements the same $r$ and $m$.)

Proposition 2 applies for any actions the firm might choose to implement. As special cases it applies (1) if the firm can implement without gainsharing an $r$ and $m$ that maximize the net of profits minus risk costs and (2) if the firm can implement without gainsharing an $r$ and $m$ that would maximize profits if there were no risk costs. Finally, we point out that Proposition 2 goes in one direction. If the firm can implement $r$ and $m$ by precluding gainsharing it can also do so by allowing gainsharing. However, if it can implement $r$ and $m$ by allowing gainsharing there is no guarantee that it can do so while precluding gainsharing. Thus. the firm can do no worse and might do better if it allows gainsharing.

We next examine two different reward systems that anticipate gainsharing. We study these reward functions because they are simple and relatively robust with respect to errors the firm might make.

\section{Two Practical Internal Customer-Internal Supplier Incentive Systems}

The interpretation of $s$ is different between the two systems we describe. We anticipate that the firm would choose whichever of the two systems that matches best its culture. In the first system 
the internal customer, Marketing, reveals its evaluation by selecting the proportion of its reward that depends upon outcomes. The remaining proportion is a fixed bonus. The (implied) evaluation by which the internal supplier, $R \& D$, is rewarded is the proportion of outcome-dependent compensation that Marketing selects. In the second system the internal customer selects a target and is rewarded by how close it gets to that target. The (implied) evaluation by which the internal supplier is rewarded is the target.

Our analysis of these reward systems is driven by the managerial problem faced by $R$ and $M$-- selecting the "right" technology. For example, Zettelmeyer and Hauser (1995) report that CEOs and CTOs are more concerned that $R$ and $M$ select the right technology than they are about minimizing risk costs. (CEOs and CTOs are concerned about risk costs and would like to keep them small but this appears to be a less critical problem than providing the incentives for the right technology.) Thus, in our analyses we focus on reward systems that provide $R$ and $M$ with the incentives to select those actions, $r^{*}$ and $m^{*}$, that would maximize the (risk neutral) firm's expected profits if it had the power and knowledge to dictate actions, observe actions, and reimburse employees only for their costly actions (as if the employees bear no risk). That is,

$$
r^{*} \text { and } m^{*} \text { maximize } \pi(r, m)-c_{M}(m)-c_{R}(r)
$$

For each of the two reward systems that we study we seek those particular $v$ 's and $w$ 's that cause $R$ and $M$ to select $r^{*}$ and $m^{*}$. (In the language of agency theory, $r^{*}$ and $m^{*}$ are called the "first-best actions.")

Although we concentrate on $r^{*}$ and $m^{*}$ we can not neglect risk costs. Because the internal customer-internal supplier systems force risk-averse employees to accept risk, the firm will have to reimburse them for accepting that risk. It will have to pay a risk penalty. Thus we compute the implied risk penalty and show how the parameters of the reward functions affect that risk penalty. The firm can then select the reward system and parameters (from the two systems we analyze) to minimize risk costs. Alternatively, it can weigh risk costs vs. the ease of implementing the reward system.

As an alternative benchmark we might seek to maximize the net of profits minus the risk penalty. (In the language of agency theory, this would be called the "second best.") The managerial tradeoff is that "second-best" reward systems are generally very complicated, even when there is only one agent (Holmstrom 1979), and there is no guarantee that they will be robust with respect to 
errors the firm might make in the selection of parameters for the reward functions. Our goal is more practical. We seek reward functions that are simple, robust, and look like those that we have observed in practice, yet implement $r^{*}$ and $m^{*}$. (For low noise our benchmark is close to optimal.)

To simplify exposition we conduct our analyses in the context of employee groups with constant (absolute) risk averse utility functions (Keeney and Raiffa 1976). That is,

$$
U_{R}=1-e^{-\rho\left(v-c_{R}-g\right)} \quad U_{M}=1-e^{-\mu\left(w-c_{M}+g\right)}
$$

\section{Variable Outcome-Based Compensation Systems}

We begin with one of the simplest specifications of $v$ and $w$, linear functions of $s$. Linear functions provide a valuable starting point (and a useful benchmark) and, in single agent problems, have proven to be robust (Holmstrom and Milgrom 1987, Lilien, Kotler and Moorthy 1992). We begin by stating the general form (Equation 7) and then derive a set of parameter values that provide incentives to $R$ and $M$ such that they select $r^{*}$ and $m^{*}$. We call this system the "variable outcomebased compensation system" because one can interpret the implicit evaluation, $s$, as indicating how much of $M$ 's bonus depends upon the (variable) incremental profit, $\check{\pi}$. Formally, the variable outcome-based compensation system is given by the following functions where $y_{o}, y_{1}, z_{o}, z_{l}$, and $z_{3}$ are constants chosen by the firm.

$$
\begin{aligned}
R: & v\left(s_{1}\right) & =y_{o}+y_{1} s_{1} \\
M: & w\left(s_{1}, \pi\right) & =z_{o}+z_{1}\left(1-s_{1}\right)+z_{3} s_{1} \check{\pi} \quad s_{1} \in[0,1]
\end{aligned}
$$

That is, after observing $r$ and anticipating $m$, Marketing selects that portion of its bonus that is based on the profit indicator. (We indicate this portion by $s_{l}$, rather than $s$ to distinguish between this system and the nonlinear system in the next subsection.) The remaining portion of $M$ 's bonus is fixed. In other words, if $s_{l}=1$ then $M$ receives its fixed bonus, $z_{0}$, plus a bonus proportional to the profit indicator, $z_{3} \check{\pi}$. On the other hand, if $s_{1}=0$, then $M$ receives only a fixed bonus, $z_{o}+z_{1}$. For intermediate $s_{l}$, the portion is determined by $s_{l}$. (We could also specify $s_{l}$ as a percentage.) Intuitively we link this implicit evaluation, $s_{l}$, to $R$ because if $R$ does its job well, then $M$ will prefer to be rewarded on the profit indicator and if $R$ does its job poorly, then $M$ will prefer the guaranteed bonus. The firm attempts to select the parameters of the functions so that $R$ and $M$ choose $r^{*}$ and $m^{*}$. (In order to participate, $R$ and $M$ are compensated for their efforts and for any risk they must 
bear.)

The variable outcome-based compensation system is a formalization of the linear reward systems, popular in marketing and in TQM, that we have seen in practice. By a linear reward system we mean a system in which both $R$ and $M$ receive a bonuses that are linear functions of the evaluation that $M$ provides. When the evaluation that $M$ provides does not have a large impact on $M$ 's compensation, $z_{1}$ and $z_{3}$ will be small. If that evaluation is an internal customer satisfaction rating, if there is some cost to $M$ in providing that rating, and if $R$ 's bonus and $M$ 's bonus are linear in $M$ 's rating, then the following proposition gives us formal tools to interpret and improve internal customer satisfaction systems.

Proposition 3 (VARIABle OUTCOME-BASED COMPENSATION). For $z_{1}$ and $y_{1}$ above critical values and for $z_{3}=1$, the variable outcome-based compensation system will give incentives to $R$ and $M$ such that, acting in their own best interests, they select $r^{*}$ and $m^{*}$.

The proof and the critical values are in the appendix. The basic idea is that if $z_{l}$ is above a critical value, then $M$, in the absence of a gainshare, will set $\hat{s}_{l}=0$. Then if $y_{l}$ is above its critical value, then $R$ has sufficient incentive to provide a gainshare to $M$ in order to obtain ${ }^{8} \tilde{s}_{l}=1$. $R$ wants to keep the gainshare as small as possible and keeping this gainshare small coincides with selecting $r^{*}$ and $m^{*}$.

By Proposition 1, there is a gainshare which inflates the evaluation from $\hat{s}_{l}=0$ to $\tilde{s}_{I}=1$. We can compute the gainshare. In addition; because Marketing and R\&D bear risk, we can compute the risk penalty that the firm must pay. To compute this penalty we recognize that, in the solution to Equation 5, the firm would only need to pay $R$ and $M$ for their "effort" costs, $c_{R}\left(r^{*}\right)$ and $c_{M}\left(m^{*}\right)$. The risk penalty is the amount by which $v+w$ exceeds the sum of these costs. With a little algebra we obtain:

$$
\begin{aligned}
g & =z_{1}-\pi^{*}+c_{M}^{*}+\mu \sigma^{2} / 2 \\
\tilde{s}_{1} & =1 \\
\text { Risk Penalty } & =\mu \sigma^{2} / 2
\end{aligned}
$$

The firm can make the gainshare very small by selecting a $z_{1}$ close to its critical value, but 
the risk penalty is not affected by $z_{l}$ and $y_{l}$. The risk penalty implied by this system is equal to that which the firm would incur by transferring all risk to $M$. (In the next subsection we investigate a system with a smaller risk penalty.)

With the parameters of Proposition 3, the optimization implies the extreme value solution, $z_{3} \tilde{s}_{l}=1$. That is, $M$ 's compensation becomes a constant plus $\check{\pi}$. Thus, in equilibrium, the linear system transfers the responsibility and rewards for the incremental outcome, $\check{\pi}$, from the firm to $M$. $M$ signals whether or not it will accept this responsibility with the evaluation, $s_{l}$, that it provides. $R$ is rewarded whenever $M$ gives an evaluation that indicates that $M$ accepts this responsibility. This gives $R$ the incentives to provide the efforts, $r$, and gainshare, $g$, so that $M$ will accept the responsibility.

Transferring responsibility to $M$ is similar in some ways to a mechanism that the agencytheory literature (e.g., Milgrom and Roberts 1992, pp. 236-239) calls "selling the firm to the agent." However, in our case $M$ becomes the residual claimant only for the incremental outcomes of $r$ and $m$ and only for this interaction. The firm retains responsibility for those outcomes (other than the measurement error) that do not depend upon $r$ and $m$. While the actions and outcomes are the same as making $M$ the residual claimant for the incremental outcomes of $r$ and $m$, we have found that many managers find a linear evaluation system more reasonable than "selling the firm." The evaluation system implies incremental responsibility for incremental actions while "selling the firm," perhaps unintendedly and inadvertently, implies transferring assets, future responsibility, and future rights for global rather than incremental actions. There are likely to be other practical implementation issues not captured by the formal model. Interpreted with this perspective, the system in Equation 7 with $z_{1}$ and $z_{3}$ as given, and its interpretation as a modified internal customer satisfaction system, is a practical means to implement a profit-center-like approach. While the actions and outcomes are the same, the mechanism -- an evaluation of $R$ by $M$, appears to many managers to be more acceptable.

Thus, a linear evaluation system and a profit center system can be more or less equivalent from an agency theory perspective, but they are likely to be perceived as different systems by employees and managers. This equivalence may be a new perspective. For example, Harari (1993) argues that internal customer satisfaction systems should be abandoned and replaced with profit center systems. We have spoken to many managers who are strong advocates of internal customer- 
internal supplier systems. None have described such systems as a means to implement a profit center. From this perspective, Proposition 3 gives us:

$\checkmark \quad$ an interpretation of a linear internal customer satisfaction system (modified to make higher $s_{l}$ more costly to $M$ ) as a means to implement a profit-center system.

$\checkmark \quad$ an indication why it is important that the evaluation the internal customer selects should affect the rewards that the internal customer receives.

a baseline by which to evaluate non-linear internal customer-internal supplier systems such as the target-value system (analyzed next).

a means to transfer incremental profit responsibility for $r$ and $m$ to the internal customer with a mechanism advocated by the TQM and customer-satisfaction literatures.

The profit-sharing system is surprisingly robust. As long as the firm sets $y_{1}$ and $z_{1}$ above the critical values, it can get $r^{*}$ and $m^{*}$.

\section{Target-Value Compensation Systems}

We now introduce non-linearity into the system by making $M$ 's rewards a non-linear function of $s$. Specifically, we select a quadratic function of $s-\check{\pi}$. The linear and quadratic functions are not the only functional forms for $w$ that will yield $r^{*}$ and $m^{*}$, however they suffice to illustrate many of the principles of internal customer-internal supplier incentive systems. ${ }^{9}$ Each has a different, but practical, interpretation. Furthermore, our experience suggests that firms are more willing to use simple rather than complex functional forms in compensation systems. See also Lilien, Kotler and Moorthy (1992).

Formally, the target-value system is given by the following functions where $v_{o}, v_{l}, w_{o}$, and $w_{2}$ are constants chosen by the firm.

$$
\begin{aligned}
R: & v\left(s_{2}\right) & =v_{o}+v_{1} s_{2} \\
M: & w\left(s_{2}, \pi\right) & =w_{o}-w_{2}\left(s_{2}-\check{\pi}\right)^{2}
\end{aligned}
$$

That is, after observing $r$ and anticipating $m, M$ selects a target value, $s_{2}$, for the profit indicator, $\check{\pi}$. $M$ receives its maximum bonus if the realized profit indicator, $\check{\pi}$, equals the target and is penalized for deviations from the target. Note that the target-value function penalizes targets that are set too high and too low. We have discussed this concept with managers at commercial banks, computer 
manufacturers, imaging firms, chemical companies, oil companies, and automotive companies. In each instance they found the idea of a target-value system quite appealing. They felt that the benefit of an accurate target could outweigh concerns about penalizing an employee group for exceeding its target. The target-value concept is similar to Gonik reward functions (Gonik 1978, Mantrala and Raman 1990) used in salesforce compensation. (Gonik reward functions encourage salespeople to make accurate forecasts by penalizing them for selling more or less than the targets that they set. ${ }^{10}$ )

PROPOSITION 4 (TARget VALUE). For $v_{l}=1$ and for $0<w_{2}<1 /\left(2 \mu \sigma^{2}\right)$, the target value compensation system will give incentives to $R$ and $M$ such that, acting in their own best interests, they select $r^{*}$ and $m^{*}$.

The proof in the appendix is constructive. We first compute rewards for $R$ and $M$ that are implied by Equation 9. We use Equation 3 to compute the implied gainshare. We use this gainshare in Equation 4 to compute $R$ 's net rewards. After these substitutions, we maximize Equation 4 subject to the constraints imposed by Equation 3. This yields the equations for the goals of $R$ and $M$. We show that these goals yield the same solution as Equation 5 -- the firm's goals. Finally, we set $v_{o}$ and $w_{o}$ so that both $R$ and $M$ get sufficient rewards so that they prefer participating over not participating.

To get an intuitive feel for how the target value function works, notice that, in the absence of gainsharing, $M$ would want to minimize the expected deviation of $s_{2}$ from $\check{\pi}$ and, hence, set $s_{2}$ equal to $\pi$. Because $v_{l}=1, R$ 's rewards are then proportional to $\pi$. With a gainshare $R$ can get $M$ to increase $s_{2}$ slightly. This makes $R$ 's rewards sensitive to $M$ 's costs. When $w_{2}$ is set in the range given, $R$ 's incentives are maximized at $r^{*}$ and $m^{*}$.

It should not be surprising that we can find a family of non-linear reward functions, $v$ and $w$, that yield $r^{*}$ and $m^{*}$. There are a limited number of first-order equations implied by the firm's optimization. Many functional families will have enough parameters so that these equations can be solved. However, some simple functional families, like constant rewards, do not. Proposition 4 shows that a quadratic system, which has an intuitive interpretation in terms of targets, has sufficient parameters. General families may not be as simple or robust.

Proposition 4 is surprisingly robust. If the firm wants to set the constants to extract all of the surplus, the firm needs to know the expected profits and costs. However, if these constants can 
be set, the firm can achieve $r^{*}$ and $m^{*}$ for any $w_{2}$ within a wide range. In the formal game the firm simply sets $v_{o}, v_{l}, w_{o}, w_{2}$. In practice we might expect the firm to find the right total compensation by trial and error. This trial and error is made easier because the firm does not need trial and error on $w_{2}$ and $v_{l}$ in order to get $r^{*}$ and $m^{*}$. We return to this issue later in the paper.

Based on the parameters of Proposition 4 we compute the gainshare, the implied evaluation, and the risk penalty.

$$
\begin{aligned}
g & =c_{M}\left(m^{*}\right)+1 /\left(4 w_{2}\right)-\mu \sigma^{2} / 2 \\
\tilde{s}_{2}-\pi^{*} & =1 /\left(2 w_{2}\right)-\mu \sigma^{2} \\
\text { Risk Penalty } & =-(2 \mu)^{-1} \log \left(1-2 \mu \sigma^{2} w_{2}\right)+\left(\mu \sigma^{2} / 2\right)\left(1-2 \mu \sigma^{2} w_{2}\right)
\end{aligned}
$$

First note that when there is no noise $\left(\sigma^{2}=0\right)$, there is no risk penalty but there is still a gainshare and the reported target exceeds the amount that $M$ will achieve. (The condition on $w_{2}$ is required for other reasons, but it also assures that the gainshare exceeds $M$ 's costs and that the evaluation exceeds the target profit.)

Second, note that both the gainshare and the risk penalty depend on the firm's choice of $w_{2}$. If the firm chooses $w_{2}$ close to its upper bound, then it can make the gainshare smaller, but its risk penalty increases. Thus, for the target-value system, there is an inherent tension between the gainshare and the risk penalty. Suppose that we make $M$ 's penalty for misforecasting small $\left(w_{2} \rightarrow 0\right)$. Then, the gainshare gets large, the distortion in the evaluation $\left(s_{2}\right.$ vs. $\left.\pi\right)$ gets large, and the risk penalty approaches that which the firm would incur had it transferred all risk to $M$. (If $M$ bore all the risk, its risk premium would be $\mu \sigma^{2} / 2$.) In other words, in systems where there is only mild social pressure for $M$ to get the forecast right ( $w_{2}$ very small), selected targets will be much larger than achievable targets, gainsharing will be large, and the firm will incur a larger risk penalty.

For $\mu \sigma>1$, the risk penalty can be minimized for a $w_{2}$ between the extremes and this minimum will be less than $\mu \sigma^{2} / 2 .^{11}$ In repeated situations the firm might get to that minimum by trial and error, but in the formal game it needs to know $M$ 's risk aversion coefficient and the noise in the profit measure. For $\mu \sigma \leq 1$, the firm can still get $r^{*}$ and $m^{*}$, but the risk penalty will exceed $\mu \sigma^{2} / 2$. 


\section{Sensitivity}

Both of the incentive systems that we have examined share the property that if the firm sets the constants, $v_{o}$ and $w_{o}$ or $y_{o}$ and $z_{o}$, too low, either Marketing or R\&D (or both) will choose not to participate (if they are well informed). If the firm sets these constants too high, either Marketing or R\&D (or both) will be overpaid. This property is not unique to the systems we study in this paper. It is a property of most incentive contracts.

In the formal game the firm needs to know values such as $\pi^{*}, c_{M}{ }^{*}, c_{R}{ }^{*}, \mu, \sigma^{2}$, and $\pi\left(r^{*}, 0\right)$ in order to set the fixed components of compensation, $v_{o}$ and $w_{o}$ or $y_{o}$ and $z_{o}$. This conceptual problem is shared with all incentive systems.

However, to implement the variable outcome-based compensation system and the target-value system, the firm must do more than select the fixed components of compensation. It must also select the coefficients that determine how compensation varies as a function of the actions and evaluations. In the linear system the firm must select the relative coefficient, $z_{1} / z_{3}$, that sets the tradeoffs that $M$ must make between compensation that depends upon outcomes and compensation that is guaranteed. The firm must also set the coefficient, $y_{l}$ that determines how $R$ is rewarded based on $s$. In the target-value system the firm must select the coefficient, $w_{2}$, that penalizes $M$ for deviations from its target. (Recall $v_{l}=1$.) We now examine the sensitivity of the compensation systems to these variable parameters.

We have already shown that the variable compensation system is not sensitive to $z_{l}$ and $y_{l}$ as long as they are above their critical values and that the target value system is not sensitive to $w_{2}$ as long as it is within a reasonable range. We state these facts as corollaries for emphasis. (The proofs are obvious by recognizing the conditions of Propositions 3 and 4 . However, for completeness they are given in the appendix.) In Corollary $1, y_{l}^{o}$ and $z_{l}^{o}$ refer to the critical values in Proposition 3. In Corollary $2, w_{2}{ }^{*}$ refers to the $w_{2}$ that minimizes the risk penalty. ${ }^{12}$ The firm may have a hard time setting $w_{2}^{*}$ because it would need to know $\mu$ and $\sigma^{2}$ in order to select this value.

Corollary 1 (VARIABle Outcome-BASEd Compensation SEnsitivity). Suppose the firm makes an error and selects a value, $z_{1}{ }^{\prime}$, that is different than $z_{1}{ }^{\circ}$, or a value, $y_{1}{ }^{\prime}$, that is different than $y_{1}{ }^{\circ}$. 
Then the system still yields $r^{*}$ and $m^{*}$ as long $z_{1}^{\prime} \geq z_{1}{ }^{o}$ and $y_{1}^{\prime} \geq y_{1}^{o}$. The risk penalty is unaffected.

COROllary 2 (TARget VAlue SENSITIVITY). Suppose the firm makes an error and selects a value, $w_{2}$, that is different than the optimal value, $w_{2}{ }^{*}$. Then the target-value compensation system still yields $r^{*}$ and $m^{*}$ as long as $0<w_{2}^{\prime}<1 /\left(2 \mu \sigma^{2}\right)$. The risk penalty increases but it can be less than the risk penalty for the variable outcome-based compensation system when $\mu \sigma>1$.

\section{Commentary}

Both the variable compensation and the target-value systems have intuitive interpretations and provide incentives to R\&D and Marketing to select $r^{*}$ and $m^{*}$ even when there is gainsharing and even when $R$ and $M$ are acting in their own best interests. When we compare the two systems we see that the target-value system can be superior to the variable compensation system on the criterion of the risk penalty. (Figure 3 is an example plot of the risk penalty as a function of $w_{2}$. We have also plotted the (constant) risk penalty for the linear system. (In this plot $\mu=5$ and $\mu \sigma^{2} / 2=.25$.)

We have stated the variable compensation system as if $M$ chose a percentage and the target value system as if $M$ chose an incremental profit target. Propositions 3 and 4 are not limited to these situations. The firm could choose to implement either system by defining a rating scale such as the 5-, 7-, or 9-point Likert or semantic-anchor scale. To implement the variable compensation system the firm would have to transform the rating scale into a percent and announce this to both $R$ and $M$. To implement the target value system, the firm would have to transform the rating scale into a target. Naturally, the firm would have to scale the parameters of the reward functions to assure consistent units in the relevant equations, either Equations 7 or Equations 9. To implement the variable compensation system the upper bound of the scale, say a ' 5 ' on a 5-point scale, would correspond to $100 \%$. To implement the target value system the firm would have to select the upper bound (if any) such that the constraint is not binding on the implied optimization problems in Equations 3-4.

Proposition 3 implies an evaluation at its upper bound and Proposition 4 implies evaluations. $s_{2}$, that are inflated beyond $\pi$ by gainsharing. We have spoken to many managers who feel that internal customer satisfaction evaluations are indeed "inflated." In fact, it is not uncommon to see evaluations clustered toward the top of the scale. [Menezes (1991) gives a published example where Xerox has chosen a target (external) satisfaction that implies every customer at the top of the scale. 
We have seen many other examples in practice.] Indeed, if there is no cost to $M$ of providing a higher evaluation for $R$, then gainsharing should be easier and, when possible, the evaluations should be inflated more.

We analyze both the variable-compensation and target-value systems because, at least qualitatively, many of the internal-customer satisfaction systems that we have observed have properties similar to the formal models. The linear system is a logical first-cut, the quadratic system provides an evolution to which managers can move. In many observed systems, management begins by giving the evaluating group (Marketing) little or no penalty, $w_{2}$, for misreporting $s_{2}$. We predict that these firms could improve their systems by making the evaluator ( $M$ 's) compensation depend more steeply on the evaluation.

\section{Relationship to Other Incentive Systems}

The internal customer-internal supplier incentive systems share some properties with two commonly used evaluation and incentive systems.

One common practice is to make Marketing and R\&D "profit" centers. That is, as Marketing purchases more projects from $R \& D$, the internal "profit" of $R \& D$ increases and Marketing's performance becomes more dependent on the quality of the inputs. For example, at Chevron, the operating divisions can "purchase" projects from the R\&D division (or from outside the firm). The $R \& D$ division survives only if it sells these projects. Both of the incentive systems we analyze share the property that Marketing signals its use of an R\&D project with its choice of $s$; in the profit-center system Marketing signals its purchase of R\&D's technology with the acceptance of a transfer price. Once Marketing chooses to use that project, its variable compensation depends upon the quality of R\&D's performance. (As discussed earlier, even the risk implications are similar between a profit center and the variable compensation system.) The literature on profit centers stresses the advantages of decentralized decision making which uses the superior local knowledge of the downstream manager (Melumad, Mookherjee and Reichelstein 1992, Eccles 1983, Grabski 1985). The internal customer-internal supplier systems present an alternative way to exploit the ability of Marketing to evaluate the actions of R\&D.

Another common practice, often used in combination with other systems, is "management by objectives" (MBO). In a typical MBO system, top management consults with the marketing 
group to develop a set of objectives for use in later evaluation. For example, the marketing group might select a target sales of $\$ 5$ million and a target customer satisfaction of $90 \%$ "extremely satisfied." Note the similarity to the target-value system. In the target-value system Marketing selects a "profit" goal, $s_{2}$. However, this "profit" goal can itself be made up of profit indicators such as net sales and satisfaction. Compared to an MBO, the target-value system specifies a specific reward function and the target, $s_{2}$, is used to reward R\&D. The target-value system relies on the superior local knowledge of Marketing which gives it the ability to evaluate the actions of R\&D. This is similar to the common motivation for an MBO, that is, MBOs allow the firm to tap into superior local knowledge (Christensen 1982).

Many firms have adopted integrating mechanisms which allow Marketing and R\&D to communicate on both customer needs and technological solutions. Some of these systems include cross-functional teams (Henke, et. al 1993), quality function deployment (Griffin 1992), relocation and physical facility redesign (Allen 1986), personnel movement (Roberts 1987), informal social systems (Moore 1987), and coordinating groups (Lorsch and Lawrence 1965). To the extent that these integrating mechanisms allow Marketing to evaluate the actions of R\&D and to the extent that these systems enable gainsharing between Marketing and R\&D, they enhance the ability of the internal customer-internal supplier systems to work. These Marketing-R\&D interface systems are complementary to internal customer-internal supplier systems, not substitutes.

\section{Conclusions and Suggested Research}

There is considerable pressure to push a marketing orientation deep into the organization. In many cases this means that internal suppliers, such as R\&D, see downstream groups, such as Marketing, as their internal customers. In a variety of firms the internal customer is asked to express its evaluation of the internal supplier and the internal supplier is rewarded based on that evaluation. In this paper we have attempted to provide insight on how such systems might work. We have shown that internal customer-internal supplier systems tend to encourage gainsharing between Marketing and R\&D, but that gainsharing can be factored out by the firm without any loss of profit. With the right incentive system the firm can encourage both Marketing and R\&D to choose the same actions, $r^{*}$ and $m^{*}$, that the firm would choose if it could act directly to choose $r$ and $m$ and reimburse $R$ and $M$ only for their employee costs. However, in any incentive system the 
firm must pay for the risk that it imposes on its employees. When Marketing has a superior ability to evaluate R\&D's actions, such a risk penalty might be necessary.

We have proposed two systems which can yield $r^{*}$ and $m^{*}$. These are certainty not the only systems possible, but they are among the simplest. The target-value system, in particular, should be relatively easy to implement and, in many cases, will yield a reasonable risk penalty. Judging by our field experience, the linear system seems to be the first system that management adopts. Its simplicity is appealing. We feel that some firms can do better with target-value systems, especially when $\mu \sigma>1$. Perhaps in the future we will see more internal customer-internal supplier systems evolve in this direction.

There is certainly room for new research. We use our theory to investigate the properties that $s$ and $\check{\pi}$ should have. There are important empirical challenges in developing such measures.

Our systems may not minimize the risk costs that the employees must bear (and for which the firm must pay), but they are simple and perform fairly well for a fairly general set of functions, $\pi, c_{M}$, and $c_{R}$. An alternative research strategy is to establish that some simple $v$ and $w$ minimize the risk cost for at least some reasonable profit and cost functions. In general, more complex systems can reduce the risk penalty, but the ease of implementation and the robustness may require systematic study. (See Simester, Wernerfelt and Hauser 1995 for a related case in which Equation 7 is expanded to a discrete menu of linear functions.) We can also study more general $v$ and $w$ which yield $r^{*}$ and $m^{*}$. For example, one can replace the quadratic function with more-general, nonsymmetric, concave functions of $s-\check{\pi}$ or one can attempt to reduce the risk penalty with higher-order polynomials in $s$ and $\check{\pi}$. Another direction of research is to extend the analyses to other error distributions besides normal and other utility functions besides constantly risk averse.

Other areas of research include extensions to longer chains of employee groups, to multiple actions by R\&D and Marketing, to multiple evaluating groups, to multiple groups being evaluated, to multiple evaluations, and to cases where $r$ affects $c_{M}$ or $\sigma^{2}$ directly. We might also extend the analyses to other dyadic relationships besides those of the internal customer and the internal supplier. Empirical research might investigate the practical implications of the systems we analyze and/or the implications of gainsharing as a means to coordinate Marketing and other functions. Finally, we might elaborate the formal model to include those implementation issues that explain why many firms choose an internal customer-internal supplier system as a means to implement a profit-center-like approach. 
INTERNAL CUSTOMERS AND INTERNAL SUPPLIERS

ENDNOTES

1. Examples (in order) based on Mitsch 1990, Harari 1993, Freundlich and Schroeder 1991, Azzolini and Shillaber 1993, Personal Communication [Cable \& Wireless, Chevron, Corning, Hoechst Celanese, and Kodak], Henke, Krachenberg, Lyons 1993, and Menezes 1991.

2. Systems in which an agent selects parameters of its incentive system have been used in salesforce compensation (Basu, Lal, Srinivasan, and Staelin 1984; Lal and Srinivasan 1993; Mantrala and Raman 1990).

3. In this paper we assume normally distributed error because that enables us to derive analytical expressions for linear and quadratic reward systems. Our propositions also apply to the special case of no error. We expect that the qualitative concepts apply, at least approximately, for more general error distributions. See example approximations in Simester, Wernerfelt, and Hauser (1995).

4. Except at boundaries imposed by any external constraints imposed on $s$.

5. The choice of a scaling constant is a non-trivial practical problem. In a later section we address the sensitivity of outcomes to the choice of parameters of $v$ and $w$.

6. Indeed, the role of some perquisites, such as subsidized meals and parking, as a form of compensation is explicitly recognized by the U.S. tax code. On the other hand "quality of life" perquisites provided by internal suppliers are more difficult to tax.

7. In the special case of a constrained linear $w$ with no noise, it is possible to choose a set of parameters such that the firm maximizes profits without a gainshare. The proof is available from the authors upon request.

8. The parameters in Proposition 3 cause $M$ to report $\tilde{s}_{l}=1$. This implies a "make-or-break" situation where $R$ receives its bonus only if $M$ participates optimally. Some firms may be uncomfortable with this make-or-break situation. Under some very special conditions, the firm can 
choose an alternative parameterization such that the reported $s_{l}$ is at an intermediate value. (If the conditions do not hold, or even if they do hold, the firm could still choose the parameters in Proposition 3.) The conditions (for $r=r^{*}$ ) basically require that the risk, $\mu \sigma^{2} / 2$, be so large that $\pi-c_{M^{-}}$ risk is smaller at $m^{*}$ than $\pi-c_{M}$ is at $m=0$. That is, when Marketing's function is primarily that of a supervisor for R\&D. Even then, the reward system is not nearly as robust as that stated in Proposition 3. For the interested reader we provide a supplemental appendix stating these conditions and proving that the reward system yields $r^{*}$ and $m^{*}$. Because the proof requires that $z_{3} \tilde{s}_{1}=1$, this alternative parameterization retains the profit-center and residual claimant interpretations.

9. For the riskless case it is possible to prove Proposition 4 for any concave function of $s_{2}-\check{\pi}$ with a finite maximum.

10. Gonik reward functions use absolute deviations rather than quadratic deviations and apply to a single agent rather than to an internal customer-internal supplier dyad. By comparing the linear and quadratic systems we see that quadratic functions can provide lower risk penalties. Indeed, the Gonik absolute-value functions will share the "make-or-break" properties of the linear system.

11. For $\mu \sigma>1$, the $w_{2}$ that produces the minimum risk penalty is $\left(2 \mu \sigma^{2}\right)^{-1}\left[1-(\mu \sigma)^{-2}\right]$. For $\mu \sigma \leq 1$, the minimum occurs as $w_{2} \rightarrow 0$. An interior minimum occurs because the quadratic target value function introduces both a linear error term, $\left(1-2 \mu \sigma^{2} w_{2}\right) e$, and a quadratic error term, $-w_{2} e^{2}$, into $M$ 's reward function. For small $w_{2}$ the linear term dominates and the error term behaves as if it were $e$. For large $w_{2}$ the quadratic term dominates. For intermediate $w_{2}$ the linear term is less than $e$, but the quadratic term is not yet so large. The certainty equivalent of the sum of the two errors is a minimum. Note that the quadratic error, $e^{2}$, is proportional to a chi-square variate and hence uncorrelated with $e$.

12. For simplicity of exposition we use the word "can" in Corollary 1. More specifically, the risk penalty for the target-value system will remain below that for the variable outcome-based compensation system if $\mu \sigma>1$ and $w_{2} \in\left(0, w_{2} "\right]$ for a $w_{2}^{\prime \prime} \epsilon\left(w_{2}^{*},\left(2 \mu \sigma^{2}\right)^{-1}\right]$. Details are in the appendix. 


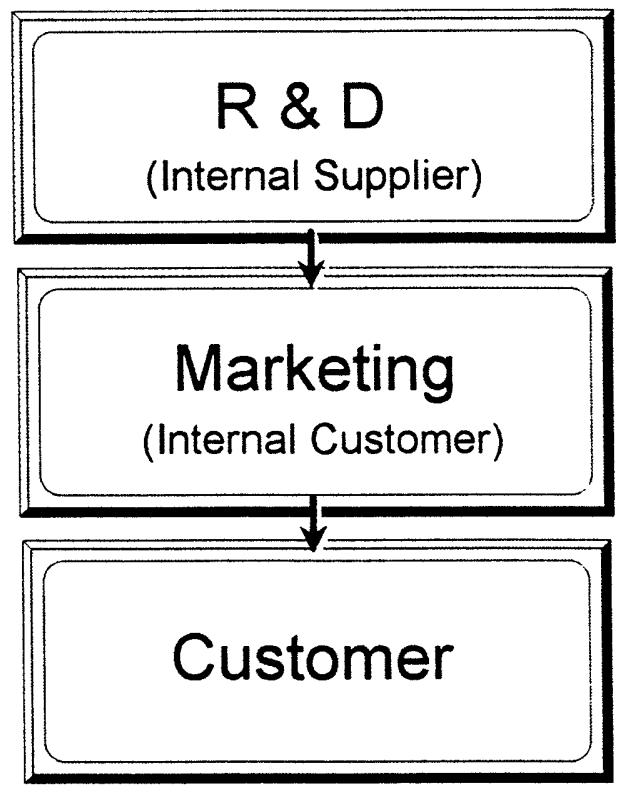

Figure 1

Conceptual Representation

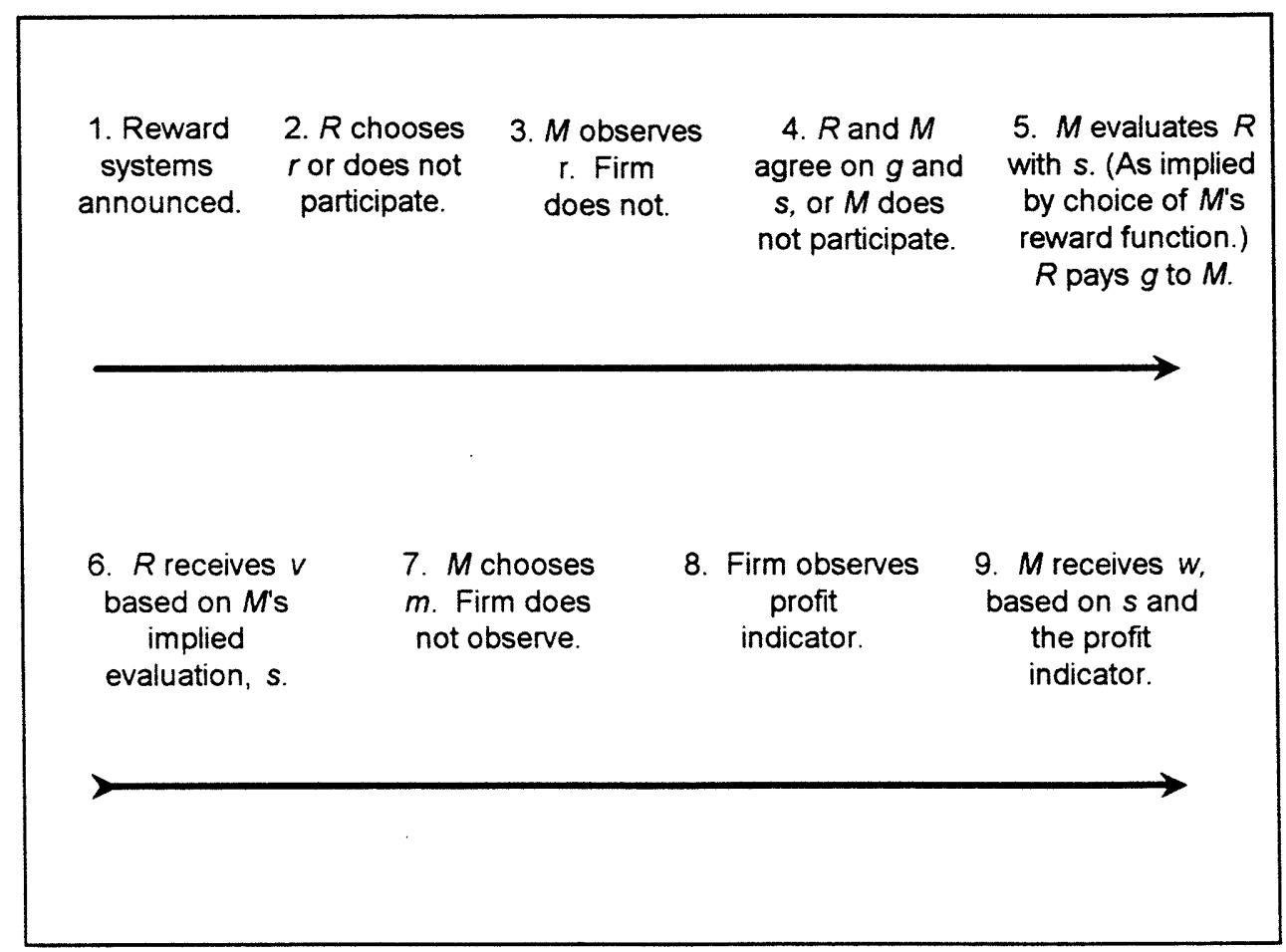

Figure 2

Order of Actions in Formal Representation

(Gainsharing Allowed.) 


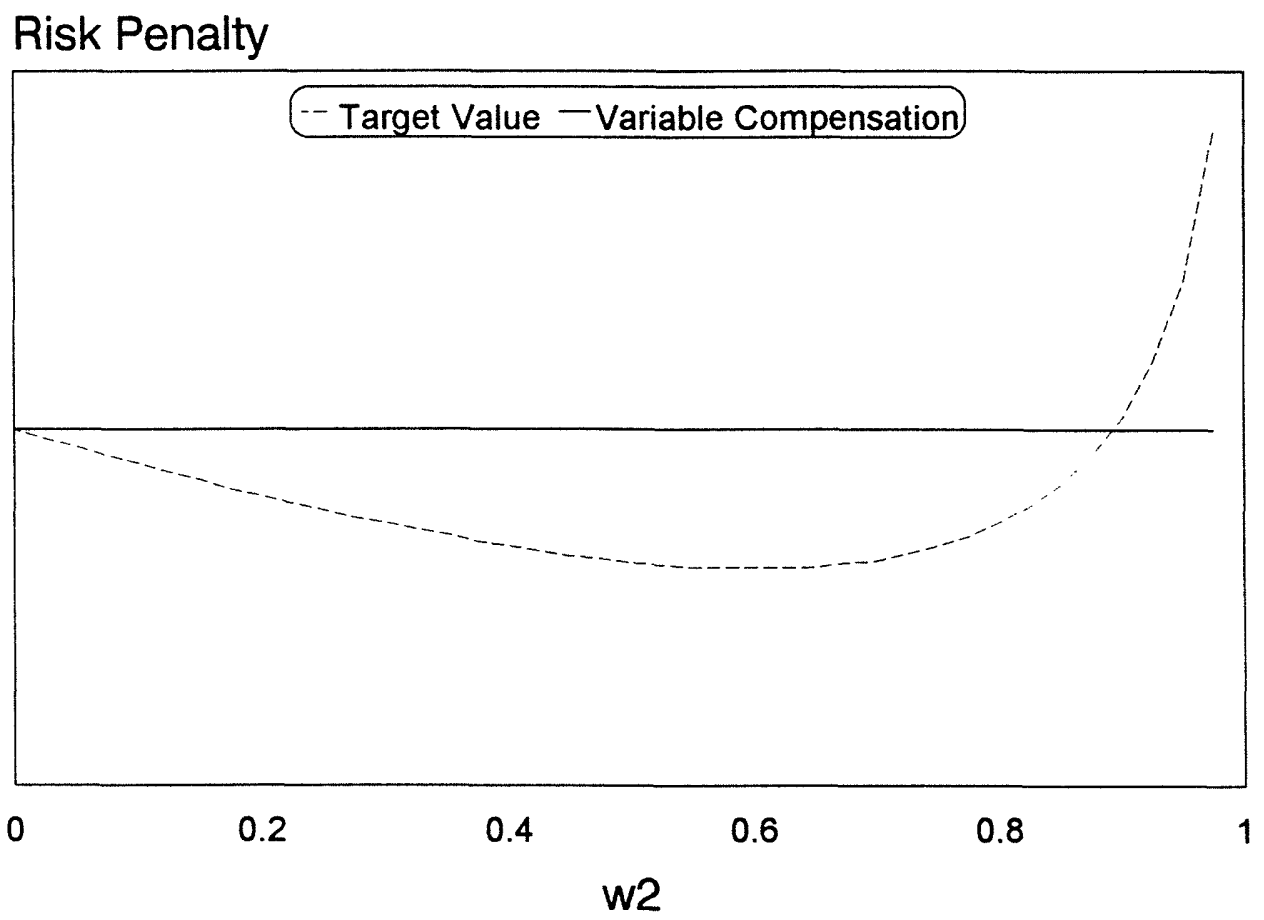

Figure 3

Risk Penalty 


\section{REFERENCES}

Allen, Thomas J. (1986), Managing the Flow of Technology, The MIT Press, Cambridge, MA.

and Ralph Katz (1992), "Age, Education, and the Technical Ladder," IEEE Transactions on Engineering Management, 39, 3, (August), 237-245.

Anderson, Eugene W., Claes Fornell, and Donald R. Lehmann (1994), "Customer Satisfaction, Market Share, and Profitability: Findings from Sweden," Journal of Marketing, 58, 3, 53-66.

Azzolini, Mary and James Shillaber (1993), "Internal Service Quality: Winning from the Inside Out," Quality Progress, 26, 11, (November), 75-78.

Basu, Amiya, Rajiv Lal, Venkataraman Srinivasan, and Richard Staelin (1984), "Salesforce Compensation Plans: An Agency Theoretic Perspective," Marketing Science, 4, 4, (Fall), 267-291.

Carder, Brooks and James D. Clark (1992), "The Theory and Practice of Employee Recognition," Quality Progress, 25, 12, (December), 25-30.

Christensen, John (1982), "Communication in Agencies," The Bell Journal of Economics, 12, 661-74.

Eccles, Robert G. (1983), "Control with Fairness in Transfer Pricing," Harvard Business Review, 61, (NovemberDecember), 149-161.

Feldman, Laurence P. (1992), "Comments on 'Motivating the Troops,'" Visions, XVI, 2, (May), 14.

Finkelman, Daniel P. and Anthony R. Goland (1990), "How Not to Satisfy Your Customers," The McKinsey Quarterly, (Winter).

Freundlich, Naomi and Michael Schroeder (1991), "Research and Development Strategies: Getting Everybody into the Act," Business Week, (October 25), 149-152.

Grabski. Severin V. (1985), "Transfer Pricing in Complex Organization: A Review and Integration of Recent Empirical and Analytical Research," Journal of Accounting Literature, 4, 33-75. 
Griffin, Abbie (1992), "Evaluating QFD's Use in US Firms as a Process for Developing Products," Journal of Product Innovation Management, 9, 3, (September), 171-187. and John R. Hauser (1994), "Integrating Mechanisms for Marketing and R\&D, " Working Paper, Marketing Science Institute, Cambridge, MA 02138.

Gonik, J. (1978), "Tie Salesmen's Bonuses to their Forecasts," Harvard Business Review, 56, 116-123.

Gouldner, Alvin W. (1965), Wildcat Strike, (New York: Harper).

Harari, Oren (1993), "Internal Customer, R.I.P.," Management Review, (June), 30-32.

Hauser, John R., Duncan I. Simester, and Birger Wernerfelt (1994), "Customer Satisfaction Incentives," Marketing Science. 13, 4, (Fall), 327-350.

Henke, John W., A. Richard Krachenberg, and Thomas F. Lyons (1993), "Cross-Functional Teams: Good Concept, Poor Implementation!," Journal of Product Innovation Management, 10, 3, (June), 216-230.

Holmstrom, Bengt and Paul Milgrom (1987), "Aggregation and Linearity in the Provision of Intertemporal Incentives, "Econometrica, 55, 2, (March), 303-320.

Keeney, Ralph L. and Howard Raiffa (1976), Decisions with Multiple Objectives: Preferences and Value Tradeoffs, (New York: John Wiley and Sons)

Kern, Jill Phelps (1993), "Toward Total Quality Management," Quality Progress, 26, 1, (January), 39-42.

Lal, Rajiv and Venkataraman Srinivasan (1993), "Compensation Plans for Single- and Multi-product Salesforces: An Application of the Holmstrom-Milgrom Model," Management Science, 39, 7, (July), 777-793.

Lilien, Gary L., Philip Kotler and K. Sridhar Moorthy (1992), Marketing Models, (Englewood Cliffs, NJ: Prentice Hall).

Lorsch. Jay W., and Paul R. Lawrence (1965), "Organizing for Product Innovation," Harvard Business Review, 109-120, (January-February). 
Mantrala, Murali K. and Kalyan Raman (1990), "Analysis of a Sales Force Incentive Plan for Accurate Sales Forecasting and Performance," International Journal of Research in Marketing, 7, 189-202.

Marketing Science Institute (1992-1994, 1994-1996), Marketing Science Institute Research Priorities: A Guide to MSI Programs and Procedures, Cambridge, MA 02138.

McDonough III, Edward F. and Richard P. Leifer (1986), "Effective Control of New Product Projects: The Interaction of Organization Culture and Project Leadership, " Journal of Product Innovation Management, 3, 3, (September), 149-157.

McLaurin, Donald L. and Shareen Bell (1993), "Making Customer Service More than Just a Slogan," Quality Progress, 26, 11, (November), 35-39.

Melumad, Nahum, Dilip Mookherjee, and Stefan Reichelstein (1992), "A Theory of Responsibility Centers," Journal of Accounting and Economics, 15, 445-84.

Menezes, Melvyn A. J. (1991), "Xerox Corporation: The Customer Satisfaction Program," Harvard Business School Case N9-591-055, (Boston, MA: Publishing Division, Harvard Business School).

Milgrom, Paul and John Roberts (1992), Economics Organization and Management, (Englewood Cliffs, NJ: Prentice Hall).

Mitsch, Ronald A. (1990), "Three Roads to Innovation," Journal of Business Strategy, 11, (September/October), 18-21.

Moore, William L. (1987), "New Product Development Practices of Industrial Marketers," Journal of Product Innovation Management, 4, 6-20.

Richardson, Peter R. (1985), "Managing Research and Development for Results," Journal of Product Innovation Management, 2, 2, (June), 75-87.

Roberts, Edward B. (1987), "Managing Technological Innovations: A Search for Generalizations," in Edward B. Roberts, ed., Managing Technological Innovation, Oxford University Press, Oxford, England.

Sidrys, Raimundas Vytenis and Inga Jakštaite (1994), "A Survey of Student Attitudes Towards Native and Foreign 
INTERNAL CUSTOMERS AND INTERNAL SUPPLIERS Instructors at Lithuanian Universities, "Lituanus, 40, 4, (Winter), 5-24.

Simester, Duncan I., Birger Wernerfelt and John R. Hauser (1994), "Overcoming Collusion: Using a Supervisor to Costlessly Resolve Moral Hazard," Working Paper, International Center for Research on the Management of Technology, MIT, Cambridge, MA 02142.

Starcher, Ronald (1992), "Mismatched Management Techniques," Quality Progress, 25, 12, (December), 49-52.

Tirole, Jean (1986), "Hierarchies and Bureaucracies: On the role of Collusion in Organization," Journal of Law, Economics, and Organization, 2, 181-214.

Zettelmeyer, Florian and John R. Hauser (1995), "Metrics to Value R\&D: Interviews with CEOs and CTOs," Working Paper, International Center for Research on the Management of Technology, MIT, Cambridge, MA, 02142.

Zeithaml, Valarie A., A. Parasuraman, and Leonard L. Berry (1990), Delivering Quality Service: Balancing Customer Perceptions and Expectations, (New York, NY: The Free Press). 
Appendix: Proofs To Propositions and Corollaries (June 1995)

PROPOSITION 1 (TEMPTATION TO GAINSHARE). For incentive systems in which the rewards to the internal supplier are increasing in the internal customer's evaluation, the internal supplier will be tempted to provide a positive gainshare to the internal customer (unless the firm sets a binding upper bound on the evaluation). Even with a binding upper bound, there may be temptation to gainshare.

Proof. Consider first the case where there is no upper bound on $s$ or it is not binding. $R$ will seek to maximize the expression in Equation 4 subject to the conditions imposed by $M$ 's maximization problems in Equation 3. Define $\Gamma_{R}=v-c_{R^{-}}-g$, then $R$ maximizes $E U_{R}$ (Equation 4) which implies

$$
\frac{\partial E U_{R}}{\partial \Gamma_{R}} \frac{\partial \Gamma_{R}}{\partial v} \frac{\partial v}{\partial \tilde{s}}+\frac{\partial E U_{R}}{\partial \Gamma_{R}} \frac{\partial \Gamma_{R}}{\partial g} \frac{\partial g}{\partial \tilde{s}}=0
$$

However, $R$ must recognize that changing $\tilde{s}$ causes $M$ to readjust its maximizations defined by Equation 3. Let $\Gamma_{M}=w-c_{M}+g$ be the argument of the expected utility operator. Implicit differentiation of Equation 3 implies ( $\tilde{s}$ does not affect the RHS of Equation 3):

$$
\begin{aligned}
\frac{\partial E U_{M}}{\partial \Gamma_{M}} \frac{\partial \Gamma_{M}}{\partial w} \frac{\partial w}{\partial \tilde{s}} & +\frac{\partial E U_{M}}{\partial \Gamma_{M}} \frac{\partial \Gamma_{M}}{\partial g} \frac{\partial g}{\partial \tilde{s}} \\
& +\frac{\partial E U_{M}}{\partial \Gamma_{M}} \frac{\partial \Gamma_{M}}{\partial w} \frac{\partial w}{\partial \pi} \frac{\partial \pi}{\partial \tilde{m}} \frac{\partial \tilde{m}}{\partial \tilde{s}}+\frac{\partial E U_{M}}{\partial \Gamma_{M}} \frac{\partial \Gamma_{M}}{\partial c_{M}} \frac{\partial c_{M}}{\partial \tilde{m}} \frac{\partial \tilde{m}}{\partial \tilde{s}}=0
\end{aligned}
$$

By recognizing that $\partial \Gamma_{M} / \partial w=\partial \Gamma_{M} / \partial g=-\partial \Gamma_{M} / \partial c_{M}=1$, and by recognizing that the last two terms vanish by the envelope theorem, this Equation reduces to:

$$
\frac{\partial E U_{M}}{\partial \Gamma_{M}} \frac{\partial w}{\partial \tilde{s}}+\frac{\partial E U_{M}}{\partial \Gamma_{M}} \frac{\partial g}{\partial \tilde{s}}=0
$$

Solving A2 for $\partial g / \partial \tilde{s}$, substituting the expression in A1, and recognizing that $\partial \Gamma_{R} / \partial v=-\partial \Gamma_{R} / \partial g=1$ yields:

$$
\frac{\partial v}{\partial \tilde{s}}=-\left(\frac{\partial E U_{R}}{\partial \Gamma_{R}}\right)^{-1}\left(\frac{\partial E U_{M}}{\partial \Gamma_{M}}\right)^{-1}\left(\frac{\partial E U_{R}}{\partial \Gamma_{R}}\right)\left(\frac{\partial E U_{M}}{\partial \Gamma_{M}}\right)\left(\frac{\partial w}{\partial \tilde{s}}\right)
$$

Thus, R's maximization problem implies $\partial v / \partial \tilde{s}=-\partial w / \partial \tilde{s}$. By assumption $v$ is an increasing function of $s$, therefore $\partial v / \partial \tilde{s}>0$. Hence, A3 implies $\partial w / \partial \tilde{s}<0$. However, without gainsharing $M$ maximizes the RHS of equation 3. This implies $\partial w / \partial \hat{s}=0$. Thus, $\tilde{s} \neq \hat{s}$. To prove $\tilde{s}>\hat{s}$, assume the opposite. If $\tilde{s}<\hat{s}$, then $\partial v / \partial \hat{s}>0$ implies $v(\tilde{s})-c_{R}(\tilde{r})-g(\tilde{r}, \tilde{s})<v(\hat{s})-c_{R}(\tilde{r})-O$. This contradicts the optimality of $\tilde{s}$, thus $\tilde{s}>\hat{s}$. Thus, the gainshare is positive. In the case where $s$ is constrained, we add a Lagrange multiplier, $-\lambda\left(s-s_{\text {max }}\right)$, to $R$ 's optimization problem. This might allow a solution of the form $\partial v / \partial \tilde{s}=\lambda$ and $\partial w / \partial \tilde{s}$ $=0$. The variable outcome-based compensation reward system gives an example where one can achieve the $r^{*}$ and $m^{*}$ with a binding constraint and a gainshare. See Proposition 3.

Benchmark. For interior solutions, our benchmark. Equation 5, implies that $r^{*}$ and $m^{*}$ solve the following first order conditions. Concave $\pi$ and convex $c_{R}, c_{M}$, imply a global maximum. 


$$
\frac{\partial \pi}{\partial r}=\frac{\partial c_{R}}{\partial r} \text { and } \frac{\partial \pi}{\partial m}=\frac{\partial c_{M}}{\partial m}
$$

PROPOSITION 2 (GAINSHARING DOESN'T HURT PROFITS). If the firm can preclude gainsharing and design a reward system, $v$ and $w$, to implement $r$ and $m$, then, without any loss of profits, the firm can design a reward system that allows gainsharing and implements the same $r$ and $m$.

Proof. We prove the theorem for any implementable actions, $r^{\circ}$ and $m^{\circ}$. Hence, it holds for the special case of $r^{o}=r^{*}$ and $m^{o}=m^{*}$. Let $\nu^{\rho}(s)$ and $\mathrm{w}^{o}(s, \check{\pi})$ implement $r^{o}$ and $m^{o}$ without gainsharing. $\left(\pi^{o}, c_{M}^{o}\right.$ are shorthand for $\pi\left(r^{\rho}, m^{o}\right)$ and $c_{M}\left(m^{o}\right)$, respectively. Define $\Gamma_{\mathrm{M}}{ }^{o}=w^{o}(s, \check{\pi})-c_{M}\left(m^{o}\right)$ and $\Gamma_{R}^{o}=v^{\rho}(s)-c_{R}\left(r^{o}\right)$.) Then $\mathrm{s}^{\circ}, m^{o}$, and $r^{o}$ satisfy the following first order conditions.

$$
\begin{aligned}
& \frac{\partial E U_{M}}{\partial s}=\frac{\partial E U_{M}}{\partial \Gamma_{M}^{o}} \frac{\partial \Gamma_{M}^{o}}{\partial w^{o}} \frac{\partial w^{o}\left(s^{o}, \pi^{o}\right)}{\partial s}=0 \\
& \frac{\partial E U_{M}}{\partial m}=\frac{\partial E U_{M}}{\partial \Gamma_{M}^{o}}\left[\frac{\partial \Gamma_{M}^{o}}{\partial w^{o}} \frac{\partial w^{o}\left(s^{o}, \pi^{o}\right)}{\partial \pi} \frac{\partial \pi\left(r^{o}, m^{o}\right)}{\partial m}+\frac{\partial \Gamma_{M}^{o}}{\partial c_{M}} \frac{\partial c_{M}\left(m^{o}\right)}{\partial m}\right]=0 \\
& \frac{\partial E U_{R}}{\partial r}=\frac{\partial E U_{R}}{\partial \Gamma_{R}^{o}}\left[\frac{\partial \Gamma_{R}^{o}}{\partial v^{o}} \frac{\partial v^{o}\left(s^{o}\right)}{\partial s} \frac{\partial s}{\partial r}+\frac{\partial \Gamma_{R}^{o}}{\partial c_{R}} \frac{\partial c_{R}\left(r^{o}\right)}{\partial r}\right]=0
\end{aligned}
$$

These reduce to:

$$
\begin{gathered}
\frac{\partial w^{o}\left(s^{o}, \pi^{o}\right)}{\partial s}=0 \\
\frac{\partial w^{o}\left(s^{o}, \pi^{o}\right)}{\partial \pi} \frac{\partial \pi\left(r^{o}, m^{o}\right)}{\partial m}-\frac{\partial c_{M}\left(m^{o}\right)}{\partial m}=0 \\
\frac{\partial v^{o}\left(s^{o}\right)}{\partial s} \frac{\partial s}{\partial r}-\frac{\partial c_{R}\left(r^{o}\right)}{\partial r}=0
\end{gathered}
$$

Now allow gainsharing and select new reward functions, $w(s, \pi)=w^{\rho}(s, \pi)-\alpha s$ and $v(s)=\alpha s$. Recall that the firm must reimburse $R$ and $M$ for their risk costs because $\check{\pi}$ contains noise and both $R$ and $M$ are risk averse. Under the specified reward system (for no gainsharing) only $M$ incurs risk due to $w^{\rho}(s, \check{\pi})$. Because the noise does not affect $s$, the only risk that $M$ will incur under the new reward system is still due to $w^{o}(s, \check{\pi})$. Thus, if the new reward system implements $\nu^{o}, m^{o}, s^{o}$, then the cost of risk will be the same for $M$ and hence for the firm which must reimburse $M$ for that risk. We must only prove that an $\alpha$ can be chosen such that the new reward functions implement $r^{\circ}, m^{o}$, and $s^{o}$ when gainsharing is allowed.

For a given $r^{\circ}$, without a gainshare. $M$ would maximize the right hand side (RHS) of Equation 3. After simplification similar to that used to derive Equations A5-A7, $\hat{m}\left(r^{0}\right)$ and $\hat{s}\left(r^{\circ}\right)$ are defined by the following. ( $\hat{\pi}$ is shorthand for $\pi\left(r^{0}, \hat{m}\right)$.) 


$$
\begin{gathered}
\frac{\partial w^{o}(\hat{s}, \hat{\pi})}{\partial s}-\alpha=0 \\
\frac{\partial w^{o}(\hat{s}, \hat{\pi})}{\partial \pi} \frac{\partial \pi\left(r^{\circ}, \hat{m}\right)}{\partial m}-\frac{\partial c_{M}(\hat{m})}{\partial m}=0
\end{gathered}
$$

For a given $r^{\rho}$, with a gainshare contract $(g, \tilde{s}), M$ will choose $\tilde{m}$ to maximize the left hand side (LHS) of Equation 3. After simplifying we obtain $(\tilde{\pi}$ is shorthand for $\pi(\tilde{r}, \tilde{m})$.)

$$
\frac{\partial w^{0}(\tilde{s}, \tilde{\pi})}{\partial \pi} \frac{\partial \pi(\tilde{r}, \tilde{m})}{\partial m}-\frac{\partial c_{M}(\tilde{m})}{\partial m}=0
$$

If $\tilde{r}=r^{\circ}$ and $\hat{s}=s^{o}$, then Equation A10 is the same as Equation A6 implying $\tilde{m}=m^{o}$.

Now $R$ chooses $\tilde{s}, \tilde{r}$, and $g$ to maximize Equation 4 subject to Equation 3 . Use the definition of $\Gamma_{M}$ and $\Gamma_{R}$ from the proof to Proposition 1. We first differentiate Equation 4 and simplify.

$$
\begin{gathered}
\alpha-\frac{\partial g}{\partial s}=0 \\
-\frac{\partial c_{R}(\tilde{r})}{\partial r}-\frac{\partial g}{\partial r}=0
\end{gathered}
$$

We use implicit differentiation on Equation 3. (The RHS is not a function of $\tilde{s}$.) After simplification:

$$
\frac{\partial w^{o}(\tilde{s}, \tilde{\pi})}{\partial s}-\alpha+\frac{\partial g}{\partial s}=0
$$

Substituting Equation A11 into Equation A13 yields Equation A5 thus if $\tilde{r}=r^{\circ}$, then $\tilde{s}=s^{0}$ because $\tilde{m}=m^{o}$ whenever $\tilde{r}=r^{\circ}$ and $\tilde{s}=s^{o}$. We must now show that we can choose $\alpha$ such that $\tilde{r}=r^{\circ}$.

We begin by implicitly differentiating Equation 3 with respect to $r$. After extensive simplification this becomes Equation A14. (We use the first-order conditions in Equations A5, A6, A8, and A9 to eliminate many terms.)

$$
\frac{\partial E U_{M}}{\partial \Gamma_{M}}\left[\frac{\partial w^{o}(\tilde{\mathcal{S}}, \tilde{\pi})}{\partial \pi} \frac{\partial \pi(\tilde{r}, \tilde{m})}{\partial r}+\frac{\partial g}{\partial r}\right]=\frac{\partial E U_{M}}{\partial \Gamma_{M}^{o}}\left[\frac{\partial w^{o}(\hat{s}, \hat{\pi})}{\partial \pi} \frac{\partial \pi(\tilde{r}, \hat{m})}{\partial r}\right]
$$

From Equation A12 we see that $\partial g / \partial r=-\partial c_{R}(\tilde{r}) / \partial r$, hence Equation A14 will imply Equation A7 if $\partial g / \partial r=-\left(\partial \nu^{\nu}\left(s^{\circ}\right) / \partial s\right)(\partial s / \partial r)$ at $\tilde{r}=r^{\circ}$. Thus. we must choose an $\alpha$ which, by Equations A8 and A9, implies $\hat{s}, \hat{m}$, and $\hat{\pi}$, such that the following equation holds: 


$$
\frac{\partial w^{o}(\hat{s}, \hat{\pi})}{\partial \pi} \frac{\partial \pi\left(r^{o}, \hat{m}\right)}{\partial r}=\left(\frac{\partial E U_{M}}{\partial \Gamma_{M}^{o}}\right)^{-1}\left(\frac{\partial E U_{M}}{\partial \Gamma_{M}}\right)\left[\frac{\partial w^{o}\left(s^{o}, \pi^{o}\right)}{\partial \pi} \frac{\partial \pi\left(r^{o}, m^{o}\right)}{\partial r}-\frac{\partial v^{o}\left(s^{o}\right)}{\partial s} \frac{\partial s\left(r^{o}\right)}{\partial r}\right]
$$

We notice that the RHS of A15 is a constant implied by the non-gainshare reward system. Call this constant $K$. Equation A15 must hold subject to Equations A8 and A9, thus we use Equation A9 to simplify Equation A15. This yields:

$$
\frac{\partial c_{M}(\hat{m})}{\partial m} \frac{\partial \pi\left(r^{o}, \hat{m}\right)}{\partial r}-K \frac{\partial \pi\left(r^{o}, \hat{m}\right)}{\partial m}=0
$$

For a given $r^{*}$, Equation A16 can be solved for an action, $\hat{m}^{*}$. Thus, Equation A15 will imply Equation A7 if we can choose an $\alpha$ in Equation A8 such that:

$$
\alpha=\frac{\partial w^{0}\left[\hat{s}(\alpha), \pi\left(r^{0}, \hat{m}^{*}\right)\right]}{\partial s} \equiv G(\alpha)
$$

We write $\hat{s}(\alpha)$ because for any $\alpha$, the first-order conditions in Equation A8 and A9 imply an $\hat{s}$ and $\hat{m}$. In general, the implied $\hat{m}$ differs from $\hat{m}^{*}$. We must solve Equation A17 so that they are equal. Equation A17 is one equation in one unknown; it will have a solution if $d G d \alpha>1$.

To prove $\partial G \partial \alpha>1$ we need some notation. Let $h(s, m)=w^{o}\left[s, \pi\left(r^{0}, m\right)\right]-c_{M}(m)$ be the function that $M$ maximizes. By assumption $v^{\rho}(s)$ and $w^{\rho}(s, \pi)$ implement maxima, hence the second order conditions on $h(s, m)$ are satisfied globally because $h(s, m)$ is concave by the assumptions of the text. Let $a, b$, and $d$ be the elements of the Hessian, that is,

$$
\text { Hessian }=\left(\begin{array}{ll}
a & b \\
b & d
\end{array}\right)
$$

where $a=\partial^{2} h / \partial s^{2}, b=\partial^{2} h / \partial s \partial m$, and $d=\partial^{2} h / \partial m^{2}$. The second-order conditions imply $a d>0, a d-b^{2}>0$. Furthermore $b=\left(\partial^{2} w / \partial s \partial \pi\right)(\partial \pi / \partial m)$ does not vanish as $\alpha \rightarrow \infty$. Holding $\hat{m}^{*}$ and $r^{*}$ constant we compute $d G / d \alpha=\left(\partial^{2} w_{\mathrm{o}} / \partial s^{2}\right)(\partial \hat{s}(\alpha) / \partial \alpha)=a(\partial \hat{s}(\alpha) / \partial \alpha)$ and we use the implicit function theorem to compute $\partial \hat{s} / \partial \alpha=d /\left(a d-b^{2}\right)$. Hence, $d G / d \alpha=a d /\left(a d-b^{2}\right)$ which is greater than 1 whenever $a d>0, a d-b^{2}>0$ and $b^{2} \neq 0$. (Which we have just proven.)

To summarize, we have proven that an $\alpha>0$ exists such that Equation A17 is satisfied. Thus, a reward system exists such that $\hat{m}^{*}$ can be implemented. This implies $\hat{m}^{*}$ solves Equation A15 which. in turn, implies Equation A7. Thus, $\tilde{r}, \tilde{m}$, and $\tilde{s}$ must satisfy Equations A5-A7 hence $\tilde{r}=r^{*}, \tilde{m}=m^{*}$, $\hat{s}=s^{*}$ for $w(s, \pi)=w^{\rho}(s, \pi)-\alpha s$, and gainsharing is allowed.

If $r^{\circ}=r^{*}$ and $m^{\circ}=m^{*}$ we can further prove that $\alpha>0$. We do this by showing $G(\alpha=0)<0$ by first recognizing that $\partial w^{\nu}\left(s^{*}, \pi^{*}\right) / \partial s=0$ by Equation A5. $\pi\left(r^{*}, \hat{m}^{*}\right)<\pi^{*}$ by the definition of optimality for $r^{*}$ and $m^{*}$, hence $\partial w^{\prime \prime}\left[s^{*}, \pi\left(r^{*}, \hat{m}^{*}\right)\right] / \partial s<0$ by $\partial^{2} w^{\nu} / \partial \pi \partial s$. For $\alpha=0$, Equations A8 and A9 imply $\hat{m}=m^{*}$ and $\hat{s}=s^{*}$ for $r=r^{*}$, thus $\hat{s}(\alpha=0)=s^{*}$. (This is true if $w^{\circ}$ and $v^{0}$ implement $\mathrm{r}^{*}$ and $\mathrm{m}^{*}$.) Thus $\partial w^{\prime \prime}\left[\hat{s}(\alpha=0), \pi\left(r^{*}, \hat{m}^{*}\right)\right] / \partial s=\partial w^{\nu}\left[s^{*}, \pi\left(r^{*}, \hat{m}^{*}\right)\right] / \partial s=G(\alpha=0)>0$. With $d G / d \alpha>1$, the solution to $\alpha=G(\alpha)$ will be positive. 
PROPOSITION 3 (VARIABle OUTCOME-BASEd COMPENSATION). For $z_{I}$ and $y_{1}$ above critical values and for $z_{3}=1$, the variable outcome-based compensation system will give incentives to $R$ and $M$ such that, acting in their own best interests, they select $r^{*}$ and $m^{*}$. The critical conditions are $z_{1}>\max \left\{\pi\left(r^{*}, 0\right), \pi^{*}-\right.$ $\left.\mu \sigma^{2} / 2-c_{M}{ }^{*}\right\}$ and $y_{1}>z_{I}-\left(\pi^{*}-c_{M}{ }^{*}-c_{R}{ }^{*}\right)+\mu \sigma^{2}$.

Proof. Define $\hat{\pi}=\pi(r, 0)$. Let $\Omega_{\mathrm{m}}$ be $M$ 's certainty equivalent (LHS of Equation 3 ) and let $\Omega_{\mathrm{R}}$ be $R$ 's certainty equivalent (Equation 4$)$. That is:

$$
\begin{aligned}
R: \Omega_{R} & =y_{o}+y_{1} s_{1}-g-c_{R} \\
M: \quad \Omega_{M} & =z_{o}+z_{1}\left(1-s_{1}\right)+z_{3} s_{1} \pi-z_{3}^{2} s_{1}^{2} \mu \sigma^{2} / 2-c_{M}+g \\
g & =z_{1} \tilde{s}_{1}-z_{3} \tilde{s}_{1} \tilde{\pi}+z_{3}^{2} \tilde{s}_{1} \mu \sigma^{2} / 2+\tilde{c}_{M}-z_{1} \hat{s}_{1}+z_{3} \hat{s}_{1} \hat{\pi}-z_{3}^{2} \hat{s}_{1}^{2} \mu \sigma^{2} / 2-\hat{c}_{M}
\end{aligned}
$$

The condition for $\hat{s}_{I}=0$ is that $\partial \Omega_{M} / \partial s_{l}<0$ when $g \equiv 0$. ( $\hat{s}_{I}=0$ implies $\hat{m}=0$ by A5.) The condition for $\tilde{s}_{I}=1$ is that $\partial \Omega_{M} / \partial s<0$ when $g$ is allowed to vary. ( $g$ is defined in A5.) For $z_{3}=1$ we differentiate to get:

$$
\begin{array}{llrl}
\text { condition } 1 & \left(\hat{s}_{1}=0\right): & -z_{1}+\hat{\pi}-\hat{s}_{1} \mu \sigma^{2}<0 \\
\text { condition } 2 & \left(\tilde{s}_{1}=1\right): & y_{1}-z_{1}+\tilde{\pi}-\tilde{s}_{1} \mu \sigma^{2}>0
\end{array}
$$

First note that both conditions hold in the neighborhood of $r^{*}, m^{*}$. Condition 1 holds because $z_{l}>\hat{\pi}$ by the statement of the proposition. Condition 2 holds because $y_{l}>z_{I}-\pi^{*}+\tilde{s}_{l} \mu \sigma^{2}$ for all $\tilde{s}_{l} \in[0,1]$ by the statement of the proposition. If conditions 1 and 2 hold, than $\tilde{s}_{1}=1$ and $\hat{s}_{I}=0$ and we get:

$$
\begin{aligned}
\Omega_{R} & =y_{0}+y_{1}-z_{1}-1 / 2 \mu \sigma^{2}+\left[\pi(\tilde{r}, \tilde{m})-c_{M}(\tilde{m})-c_{R}(\tilde{r})\right] \\
g & =z_{1}-\left[\pi(\tilde{r}, \tilde{m})-c_{M}(\tilde{m})-1 / 2 \mu \sigma^{2}\right]
\end{aligned}
$$

At $\tilde{r}=r^{*}, \tilde{m}=m^{*}$ we have $z_{1}>\pi(\tilde{r}, \tilde{m})-c_{M}(\tilde{r})-\mu \sigma^{2} / 2$ by the statement of the proposition. Thus, $g>0$ and the maximization of $\Omega_{R}$ yields $r^{*}, m^{*}$. This means that if conditions 1 and 2 hold, which they do for $r^{*}$ and $m^{*}$, then $R$ and $M$ will choose $r^{*}$ and $m^{*}$. Furthermore, we can choose $y_{o}$ such that $R$ will participate and we can choose $\mathrm{z}_{0}$ such that $M$ will participate. We need only establish that $R$ will never choose $r$, and the implied $m$, such that conditions 1 and 2 are violated.

Suppose condition 1 is false, but condition 2 is true. Then, $\hat{s}_{1}=1, \tilde{s}_{1}=1$, and $g=0$. This implies that $\Omega_{R}=y_{0}+y_{1}-c_{R}(r)$. But condition 1 false implies that $-z_{1}+\pi(r, 0)-\mu \sigma^{2}>0$. Adding this positive number to $\Omega_{R}$ yields $\Omega_{R}<y_{o}+y_{I}-c_{R}(r)-z_{1}+\pi(r, 0)-\mu \sigma^{2}=y_{o}+y_{I}-z_{I}-\mu \sigma^{2} / 2+\left[\pi(r, 0)-c_{R}(r)\right.$ $\left.c_{M}(0)\right]-\mu \sigma^{2} / 2<y_{0}+y_{1}-z_{I}-\mu \sigma^{2} / 2+\left[\pi\left(r^{*}, m^{*}\right)-c_{R}\left(r^{*}\right)-c_{M}\left(m^{*}\right)\right]$ which is what $R$ can get with $r^{*}$ and $m^{*}$. The last inequality is by the definition of the optimal. Recall $c_{M}(0)=0$. Thus, $R$ can do better if it chooses an $r$ such that condition 1 is true than it can if condition 1 is false.

Suppose condition 2 is false. (Condition 1 can be true or false.) Then, $\tilde{s}_{l}=0, g=0$, and $\Omega_{R}=y_{0}$. This is less than $R$ can get at $r^{*}, m^{*}$. (Notice in Equation A6 that $\Omega_{R}>y_{o}$ by the definitions of $y_{1}$ and $z_{1}$ in the statement of the proposition.) Thus, $R$ can do better if condition 2 is true than if condition 2 is false.

Summarizing. $R$ can choose and prefers to choose $r$ such that conditions 1 and 2 are true. When these conditions are true, $r=r^{*}, m=m^{*}$, and $\tilde{s}_{1}=1$. 
PROPOSITION 4 (TARGET VALUE). For $v_{1}=1$ and for $0<w_{2}<1 /\left(2 \mu \sigma^{2}\right)$, the target value compensation system will give incentives to $R$ and $M$ such that, acting in their own best interests, they select $r^{*}$ and $m^{*}$.

Proof. In a supplemental appendix we demonstrate that for constantly risk averse utility and for normally distributed noise the certainty equivalent, $c$. e., of $E U_{M}\left(w-c_{M}+g\right)$ is given by the following $\left(w_{2}<\right.$ $\left(2 \mu \sigma^{2}\right)^{-1}$ assures that the logarithm is defined.)

$$
\begin{aligned}
& c . e .=\left[w_{0}\right.\left.+(1 /[2 \mu]) \log \left(1-2 \mu \sigma^{2} w_{2}\right)\right]-w_{2}\left(s_{2}-\pi\right)^{2} \\
&-2 \mu \sigma^{2} w_{2}^{2}\left(1-2 \mu \sigma^{2} w_{2}\right)^{-1}\left(s_{2}-\pi\right)^{2}-c_{M}+g \\
& \equiv w_{0}{ }^{\prime}-w_{2}{ }^{\prime}\left(s_{2}-\pi\right)^{2}-c_{M}+g
\end{aligned}
$$

Because $E U_{R}$ and $E U_{\mathrm{M}}$ are increasing transformations of the certainly equivalents, we evaluate Equations 3 and 4 in terms of certainly equivalents. Rewriting Equation 3 yields.

$$
w_{0}^{\prime}-w_{2}^{\prime}\left[\tilde{s}_{2}-\pi(r, \tilde{m})\right]^{2}-c_{M}(\tilde{m})+g=w_{0}^{\prime}-w_{2}^{\prime}\left[\hat{s}_{2}-\pi(r, \hat{m})\right]^{2}-c_{M}(\hat{m})
$$

Because $\hat{s}_{2}$ maximizes the $R H S$ of A5, it is easy to see that $\hat{s}_{2}=\pi(r, \hat{m})$ when $w_{2}>0$. Similarly we show $\hat{m}=0$. Thus,

$$
g=w_{2}^{\prime}\left[\tilde{s}_{2}-\pi(r, \tilde{m})\right]^{2}+c_{M}(\tilde{m})
$$

Since $R$ receives its reward based on $\tilde{s}_{2}$, which is reported prior to market outcomes, $\check{\pi}$, there is no risk adjustment for $R$. Hence, we incorporate $M$ 's maximization problems, A7, into $R$ 's maximization problem, Equation 4 , by substituting for $g$. Thus, the maximization problem in Equation 4 becomes:

$$
\text { maximize }\left\{v_{0}+v_{1} \tilde{s}_{2}-c_{R}(\tilde{r})-w_{2}^{\prime}\left[\tilde{s}_{2}-\pi(\tilde{r}, \tilde{m})\right]^{2}-c_{M}(\tilde{m})\right\}
$$

The first order conditions for $\tilde{m}, \tilde{r}$, and $\tilde{s}_{2}$ are given, respectively, by (A8)-(A10). ( $\tilde{\pi}, \tilde{c}_{M}$, and $\tilde{c}_{R}$ are shorthand for $\pi(\tilde{r}, \tilde{m}), c_{M}(\tilde{m}), c_{R}(\tilde{r})$ respectively.)

$$
\begin{aligned}
2 w_{2}^{\prime}\left(\tilde{s}_{2}-\tilde{\pi}\right) \frac{\partial \tilde{\pi}}{\partial m}-\frac{\partial \tilde{c}_{M}}{\partial m} & =0 \\
2 w_{2}^{\prime}\left(\tilde{s}_{2}-\tilde{\pi}\right) \frac{\partial \tilde{\pi}}{\partial r}-\frac{\partial \tilde{c}_{R}}{\partial r} & =0 \\
v_{1}-2 w_{2}^{\prime}\left(\tilde{s}_{2}-\tilde{\pi}\right) & =0
\end{aligned}
$$

By setting $v_{1}=1$, (A10) becomes $2 w_{2}^{\prime}[\tilde{s}-\pi(\tilde{r}, \tilde{m})]=1$. By substituting this relationship in (A8) and (A9), we get (A4). Finally, we select the constants, $v_{0}$ and $w_{0}$, such that $M$ 's c.e. and $R$ 's maximum are preferred to non-participation. The firm will participate if the profit in Equation 5 exceeds the risk penalty implied by $M$ 's c.e. Note that different $v_{l}$ 's implement different actions. 
COROllary 1 (VARIABle OUTCOME-BASEd COMPENSATION SENSITIVITY). Suppose the firm makes an error and selects a value, $z_{l}$, that is different than $z_{l}{ }^{\circ}$, or a value, $y_{1}$, that is different than $y_{l}{ }^{\circ}$. Then the system still yields $r^{*}$ and $m^{*}$ as long $z_{1}{ }^{\prime} \geq z_{1}^{o}$ and $y_{1}{ }^{\prime} \geq y_{1}{ }^{\circ}$. The risk penalty is unaffected.

Proof. Notice that Proposition 3 only requires that $z_{1}$ and $y_{1}$ be above their critical values. Adjusting the coefficients, $z_{o}$ and $y_{o}$ assures participation. The risk penalty in Equation 8 does not depend upon $z_{1}$ and $y_{1}$.

COROLLARY 2 (TARGET VALUE SENSITIVITY). Suppose the firm makes an error and selects a value, $w_{2}$,' that is different than the optimal value, $w_{2}{ }^{*}$. Then the target-value compensation system still yields $r^{*}$ and $m^{*}$ as long as $0<w_{2}{ }^{\prime}<1 /\left(2 \mu \sigma^{2}\right)$. The risk penalty increases but it can be less than the risk penalty for the variable outcome-based compensation system when $\mu \sigma>1$.

Proof. Notice that Proposition 4 only requires $0<w_{2}{ }^{\prime}<\left(2 \mu \sigma^{2}\right)^{-1}$. Adjusting the coefficients, $w_{0}$ and $v_{0}$, that depend on $w_{2}$ assures participation. $w_{2}{ }^{*}$ is defined as the $w_{2}$ yielding the minimum risk penalty in Equation 10, thus, by definition, the risk penalty weakly increases. Straightforward calculus applied to Equation 10 implies that the second derivative is positive, thus the increase is strict. Straightforward calculus also implies that the risk penalty approaches $\mu \sigma^{2} / 2$ as $w_{2} \rightarrow 0$, that the risk penalty approaches $\infty$ as $w_{2} \rightarrow\left(2 \mu \sigma^{2}\right)^{-1}$, and that $w_{2}{ }^{*}>0$ for $\mu \sigma>1$. (The equation for $w_{2}{ }^{*}$ is given in footnote 11.) Thus, the risk penalty will be less than $\mu \sigma^{2} / 2$ over some range, $w_{2} \in\left(0, w_{2}{ }^{\prime \prime}\right]$, where $w_{2}{ }^{*}<w_{2}{ }^{\prime \prime}<\left(2 \mu \sigma^{2}\right)^{-1}$. This implies that the risk penalty can stay less than $\mu \sigma^{2} / 2$ over this range and that the range includes $w_{2}{ }^{*}$. 
Supplemental Appendix (June 1995)

\section{Certainty Equivalent for a Squared Normal Random Variable \\ Profit-Sharing Proposition with $\tilde{s} \in(0,1)$}

LEMMA (QUADRATIC ERROR). Let $\eta$ be a zero-mean normal variable with variance $\sigma^{2}$, then, for constantly risk averse utility functions with risk parameter, $\mu$, the certainty equivalent of $\gamma+\beta \eta-w_{2} \eta^{2}$ is $\gamma+(2 \mu)^{-1} \log \left(1-2 \mu w_{2} \sigma^{2}\right)-\mu \beta^{2} \sigma^{2} /\left[2\left(1-2 \mu w_{2} \sigma^{2}\right)\right]$

Proof.

$$
\begin{aligned}
E U\left(\gamma+\beta \eta-w_{2} \eta^{2}\right) & =1-\int_{-\infty}^{+\infty} e^{-\mu \gamma-\mu \beta \eta+\mu w_{2} \eta^{2}}(\sigma \sqrt{2 \pi})^{-1} e^{-\eta^{2} /\left(2 \sigma^{2}\right)} d \eta \\
& =1-\frac{1}{\sigma \sqrt{2 \pi}} e^{-\mu \gamma} \int_{-\infty}^{+\infty} e^{-\mu \beta \eta} e^{-\eta^{2}\left[1 /\left(2 \sigma^{2}\right)-\mu w_{2}\right]} \\
& =1-\frac{\Sigma}{\sigma} e^{-\mu \gamma} \int_{-\infty}^{+\infty} e^{-\mu \beta \eta}(\Sigma \sqrt{2 \pi})^{-1} e^{-\eta^{2} /\left(2 \Sigma^{2}\right)} d \eta \\
& =1-e^{\log \Sigma-\log \sigma} e^{-\mu \gamma} e^{\mu^{2} \beta^{2} \Sigma^{2} / 2} \\
& =1-e^{-\mu\left[(1 / \mu)(\log \sigma-\log \Sigma)+\gamma-\mu \beta^{2} \Sigma^{2} / 2\right]}
\end{aligned}
$$

where $\Sigma^{2}=\sigma^{2} /\left(1-2 \mu \sigma^{2} w_{2}\right)$. The transition from the third line to the fourth line uses the $\omega$-transform, $E[\exp (-\omega \eta)]$, of a zero-mean normal distribution with variance, $\Sigma^{2}$. That is, $E[\exp (-\omega \eta)]=\exp \left(\omega^{2} \Sigma^{2} / 2\right)$. Thus,

$$
\begin{aligned}
\text { c.e. } & =\gamma+(2 \mu)^{-1}\left\{\log \sigma^{2}-\log \left[\sigma^{2} /\left(1-2 \mu \sigma^{2} w_{2}\right)\right]\right\}-\mu \beta^{2} \sigma^{2} /\left[2\left(1-2 \mu \sigma^{2} w_{2}\right)\right] \\
& =\gamma+(2 \mu)^{-1} \log \left(1-2 \mu \sigma^{2} w_{2}\right)-\mu \beta^{2} \sigma^{2} /\left[2\left(1-2 \mu \sigma^{2} w_{2}\right)\right]
\end{aligned}
$$

Substituting $\gamma=w_{o}-w_{2}(s-\pi)^{2}, \beta=2 w_{2}\left(s_{2}-\pi\right)$ gives the result used in Proposition 4.

PRoposition $3 B$ (VARIABLE OUTCOME-BASED COMPENSATION, $\tilde{s}_{l} \epsilon(0,1)$ ). The variable outcome-based compensation system yields the $r^{*}$ and $m^{*}$ for $v_{1}=z_{3}\left[\pi\left(r^{*}, 0\right)-\pi^{*}+\mu \sigma^{2}\right], z_{1}=z_{3} \pi\left(r^{*}, 0\right)$, and $z_{3}>1$ whenever $\pi^{*}-c_{M}{ }^{*}-c_{R}{ }^{*}>\mu \sigma^{2} / 2>\left[\pi^{*}-c_{M}{ }^{*}\right]-\left[\pi\left(r^{*}, 0\right)-c_{M}(0)\right]>0$ and $\mu \sigma^{2} / 2>\pi^{*} / 2-\pi\left(r^{*}, 0\right) / 2$.

Proof. Define $\hat{\pi}$ and $\Omega_{M}$ as in Proposition 3. With constant risk aversion and zero-mean normally distributed noise, the certainty equivalent, c.e., of $E U_{\mathrm{M}}\left(w-c_{M}+g\right)$ is:

$$
\Omega_{M}=z_{0}+z_{1}\left(1-s_{1}\right)+z_{3} s_{1} \pi-z_{3}^{2} s_{1}^{2} \mu \sigma^{2} / 2-c_{M}+g
$$

Because $E U_{R}$ and $E U_{m}$ are increasing transformations of the certainly equivalents, equation 3 yields. 


$$
\begin{aligned}
z_{o}+z_{1}\left(1-\tilde{s}_{1}\right) & +z_{3} \tilde{s}_{1} \tilde{\pi}-z_{3}^{2} \tilde{s}_{1}^{2} \mu \sigma^{2} / 2-c_{M}(\tilde{m})+g \\
& =z_{o}+z_{1}\left(1-\hat{s}_{1}\right)+z_{3} \hat{s}_{1} \hat{\pi}-z_{o}^{2} \hat{s}_{1}^{2} \mu \sigma^{2} c_{M}(\hat{m}) / 2
\end{aligned}
$$

Because $\hat{s}_{I}$ maximizes the RHS of SAl, we obtain $\hat{s}_{I}=\left(z_{3} \hat{\pi}-z_{I}\right) /\left(z_{3}^{2} \mu \sigma^{2}\right)$. Setting $z_{I}=z_{3} \hat{\pi}$ implies $\hat{s}_{1}=0$ which, in turn, implies $\hat{m}=0$. Rearranging SA1 yields:

$$
g=z_{3} \hat{\pi} \tilde{s}_{1}-z_{3} \tilde{\pi} \tilde{s}_{1}+z_{3}^{2} \tilde{s}_{1}^{2} \mu \sigma^{2} / 2-c_{M}(\tilde{m})
$$

The first condition on $\mu \sigma^{2} / 2$ in the statement of the Proposition assures $g>0$. Furthermore, $v_{l}>0$ by the conditions of the Proposition. The maximization problem in equation 4 then becomes:

$$
\text { maximize } \begin{aligned}
\Omega_{R}= & y_{0}+y_{1} \tilde{s}_{1}-g-c_{R}(\tilde{r}) \\
= & y_{0}+z_{3} \tilde{s}_{1} \hat{\pi}-z_{3} \tilde{s}_{1} \pi^{*}+z_{3} \tilde{s}_{1} \mu \sigma^{2}-z_{3} \hat{\pi} \tilde{s}_{1}+z_{3} \tilde{\pi} \tilde{s}_{1} \\
& \quad-z_{3}^{2} \tilde{s}_{1}^{2} \mu \sigma^{2} / 2-c_{M}(\tilde{m})-c_{R}(\tilde{r})
\end{aligned}
$$

The first-order conditions for $\tilde{m}, \tilde{r}$, and $\tilde{s}_{1}$ are given, respectively, by equations SA2-SA4.

$$
\begin{aligned}
& \left(z_{3} \tilde{s}_{1}\right) \frac{\partial \tilde{\pi}}{\partial m}-\frac{\partial \tilde{c}_{M}}{\partial m}=0 \\
& \left(z_{3} \tilde{s}_{1}\right) \frac{\partial \tilde{\pi}}{\partial r}-\frac{\partial \tilde{c}_{R}}{\partial r}=0 \\
& z_{3} \mu \sigma^{2}-z_{3}^{2} \tilde{s}_{1} \mu \sigma^{2}=0
\end{aligned}
$$

We obtain SA2 and SA4 by direct differentiation. We obtain SA3 by the chain rule recognizing that $\tilde{s}_{1}$ and $\tilde{m}$ depend on $\tilde{r}$. However, the first-order conditions for $\hat{m}$ and $\tilde{m}$ eliminate some terms. (The rest of the terms are already eliminated by $\hat{s}_{1}=0$.) By setting $z_{3} \tilde{s}_{1}=1$, SA2-SA4 reduce to equation A4 and $\tilde{s}_{1}=1 / z_{3}$. Finally, we select $y_{0}$ and $\mathrm{z}_{0}$ such that $\Omega_{m}$ and $\Omega_{R}$ are positive. The conditions of the proposition, $\pi^{*}-c_{m}{ }^{*}-c_{R}{ }^{*}-\mu \sigma^{2} / 2>0$, assure the firm participates. 